\title{
Dirac method and symplectic submanifolds in the cotangent bundle of a factorizable Lie group
}

\author{
S. Capriotti ${ }^{1}$ and $\mathrm{H}$. Montani ${ }^{2, a)}$ \\ ${ }^{1}$ Departamento de Matemática, Universidad Nacional del Sur, Av. Alem 1253, 8000 - Bahía \\ Blanca, Buenos Aires, Argentina. \\ ${ }^{2}$ Instituto Balseiro - Centro Atómico Bariloche, 8400 - S. C. de Bariloche, Rio Negro, \\ Argentina.
}

(Received 23 November 2010; accepted 2 June 2011; published online 7 July 2011)

\begin{abstract}
We study some symplectic submanifolds in the cotangent bundle of a factorizable Lie group defined by second class constraints. By applying the Dirac method, we study many issues of these spaces as fundamental Dirac brackets, symmetries, and collective dynamics. As the main application, we study integrable systems on these submanifolds as inherited from a system on the whole cotangent bundle, meeting in a natural way with the Adler-Kostant-Symes theory of integrability. (c) 2011 American Institute of Physics. [doi:10.1063/1.3603427]
\end{abstract}

\section{INTRODUCTION}

The phase spaces of many interesting dynamical systems arise as constrained submanifolds in a larger phase space. We assume that a phase space is naturally equipped with a Poisson bracket structure, thus it is a frequent problem how to restrict the Poisson algebra of functions on the larger phase space to a Poisson algebra of functions on the constrained submanifold. In the particular case in which this submanifold is the common level set of some functions which amounts to be a second class set of constraints, the Dirac method ${ }^{6}$ is a successful algorithm to produce a Poisson bracket for the constrained submanifold, the so called Poisson-Dirac or Dirac bracket. Despite the original Dirac's motivation was addressed to obtain a consistent dealing for the quantization of this kind of systems, the procedure becomes a powerful tool for working out classical constrained systems. The Dirac bracket produces Lie derivatives of functions on the whole phase space along the projection of the Hamiltonian vector fields on the tangent space of the constrained submanifold, and so it is a representation of these vectors fields in terms of the geometrical data in the total phase space. Integral curves of this projected vector field are the trajectories of the constrained Hamiltonian system.

Most of the phase space comes from configuration spaces by the Legendre transformation. In some cases, where the symmetries define many of the characteristics of the systems, configuration spaces can be identified with Lie groups, among them we can mention the rigid body and its generalizations $^{3}$ as the main finite dimensional examples, and the sigma and WZNW models in field theory being outstanding examples in infinite dimension. The corresponding phase spaces are then the cotangent bundles of these Lie groups, which can be identified with the Cartesian product of the group itself and the dual of its Lie algebra. They are symplectic manifolds, meaning that the Poisson brackets are nondegenerate, and enjoy many nice properties related to the symmetry issues. ${ }^{2,9}$ In particular, they have close relationship with integrable systems when combined with the Adler-Kostant-Symes (AKS) theory. ${ }^{1}$ This theory gives rise to nontrivial integrable systems by a restriction procedure: given a Lie algebra $\mathfrak{g}=\mathfrak{g}_{+} \oplus \mathfrak{g}_{-}$, with $\mathfrak{g}_{+}, \mathfrak{g}_{-}$Lie subalgebras of $\mathfrak{g}$, the restriction of the set of $A d$-invariant functions on a coadjoint orbit of $\mathfrak{g}$ to the coadjoint orbit of one of its components, for instance $\mathfrak{g}_{+}$, gives rise to a nontrivial set of Poisson commuting functions, so that

\footnotetext{
a) Present address: Departamento de Ciencias Exactas y Naturales, Unidad Académica Caleta Olivia, Universidad Nacional de la Patagonia Austral, Santa Cruz, Argentina. Electronic mail: hmontani@uaco.unpa.edu.ar.
} 
the Arnold-Liouville theorem holds. Further developments allow to extend these ideas to cotangent bundles of the Lie groups $G, G_{+}, G_{-}$associated with $\mathfrak{g}, \mathfrak{g}_{+}, \mathfrak{g}_{-},{ }^{11,12}$ in such a way that the solutions of the equations of motion on the cotangent bundle of the factors $G_{+}, G_{-}$arise as the $G_{+}$-factor of an exponential curve in $G$, which in turn solves an almost trivial system on the cotangent bundle of $G$.

The restriction procedures on dynamical systems associated with factorizable Lie groups and its Lie algebras strongly resembles some kind of constrained systems, and it is the main motivation of this work to encode it inside the Dirac method. In doing so, we combine all the above ingredients in the study of a family of symplectic submanifolds in the cotangent bundle of a factorizable Lie group $G=G_{+} \times G_{-}$, defined as the level set of a map which projects the cotangent bundle $T^{*} G$ onto the cotangent bundle of one of its factors. To deal with this kind of constraints we develop, in a general framework, a geometric approach to Dirac's brackets addressed to describe immersed symplectic submanifold $\mathcal{N}, l: \mathcal{N} \hookrightarrow \mathcal{M}$, as the level sets of some submersive map from $\mathcal{M}$ into another manifold $\mathcal{P}$. This approach allows for a straightforward application to the projection maps $\Psi: G \times \mathfrak{g}^{*} \longrightarrow G_{-} \times \mathfrak{g}_{-}^{*}$ and $\Upsilon: G \times \mathfrak{g}^{*} \longrightarrow G_{+} \times \mathfrak{g}_{+}^{*}$, giving rise to two family of symplectic submanifolds of $T^{*} G$, characterized as the preimages $\Psi^{-1}\left(g_{-}, \eta_{-}\right)$or $\Upsilon^{-1}\left(g_{+}, \eta_{+}\right)$, and both equipped with the corresponding Poisson-Dirac structure turning them in symplectic fibrations.

This framework allows to study many aspects of these spaces and related dynamical systems, such as symmetries and integrability. It is interesting to see how to restrict to these submanifolds the lift of the action by left translation on $T^{*} G$, revealing that an encoded crossed reciprocal action, derived from the dressing actions, ${ }^{7}$ still holds on some particular fibers. These symmetries play a central role in the application of our scheme to integrable systems, since reproducing the AKS version of Refs. 12 and 11 the solutions turn to be orbits of a factor of an exponential curve in $G .^{5}$

It seems rather natural to apply the Dirac method in the original setting of coadjoint orbits of the AKS theory; however, it is not obvious that the same happens when dealing with the fibers of $\Psi: G \times \mathfrak{g}^{*} \longrightarrow G_{-} \times \mathfrak{g}_{-}^{*}$ and $\Upsilon: G \times \mathfrak{g}^{*} \longrightarrow G_{+} \times \mathfrak{g}_{+}^{*}$. We shall see the remarkable fact that the application of our scheme to some collective models on a subfamily of these symplectic submanifolds yields projected Hamiltonian vector fields whose integral curves are generated by the action of a factor of an exponential curve in $G$ and, in turn, this exponential curve solves an almost trivial system on $T^{*} G$. This holds on the families of reduced phase spaces $\Psi^{-1}\left(g_{-}, \eta_{-}\right)$or $\Upsilon^{-1}\left(g_{+}, \eta_{+}\right)$, for $\eta_{-}$and $\eta_{+}$being stable under the coadjoint action of $G_{+}$and $G_{-}$, characters in the corresponding coadjoint representations, and they obviously include the submanifolds are $\Psi^{-1}(e, 0)=T^{*} G_{+}$and $\Upsilon^{-1}(e, 0)=T^{*} G_{-}$. Thus, the Dirac method succeeds in reproducing the procedure of factorization and restriction to one factor driving to the equations of motion of the reduced systems whose solutions are related to the factorization problem, the core of the AKS ideas. ${ }^{11}$

The relation between the submanifolds $\Psi^{-1}\left(g_{-}, \eta_{-}\right)$and $\Upsilon^{-1}\left(g_{+}, \eta_{+}\right)$in one side, and AKS systems on the other, stems from the merging of three remarkable facts.

- If $J_{-}: G \times \mathfrak{g}^{*} \rightarrow \mathfrak{g}_{-}^{*}$ is the momentum map associated with the right action of $G_{-}$on $G$ lifted to its cotangent bundle and $\eta_{-} \in \mathfrak{g}_{+}^{0}$ is a character, then every submanifold of $J_{-}^{-1}\left(\eta_{-}\right)$of the right dimension is a symplectic submanifold; ${ }^{11}$ this means that the Poisson bracket associated with this structure can be calculated as a Dirac bracket. Additionally, the canonical projection onto the reduced space $J_{-}^{-1}\left(\eta_{-}\right) / G_{-}$for this action restricts to a symplectomorphism.

- The reduced space $J_{-}^{-1}\left(\eta_{-}\right) / G_{-}$is naturally symplectomorphic to $T^{*} M$, where $M:=G / G_{-}$.

- The AKS systems can be considered as dynamical systems defined on reduced spaces associated with the lift of a particular action of $G_{+} \times G_{-}$on $G$, namely, if $J: G \times \mathfrak{g}^{*} \rightarrow \mathfrak{g}_{-}^{*} \times \mathfrak{g}_{+}^{*}$ is the momentum map for this action, then the symplectic manifold underlying AKS systems is $J^{-1}\left(\eta_{-}, \eta_{+}\right) /\left[\left(G_{-}\right)_{\eta_{-}} \times\left(G_{+}\right)_{\eta_{+}}\right]$.

From the first two items above, we see that the constrained manifolds $\Psi^{-1}\left(g_{-}, \eta_{-}\right)$are symplectic $G$-spaces, in particular $G_{+}$-spaces; if we perform reduction of any of these spaces for this action, the reduction by stages theorem ${ }^{8}$ allows us to conclude from the third item and the symplectomorphism $\Psi^{-1}\left(g_{-}, \eta_{-}\right) \simeq J_{-}\left(\eta_{-}\right) / G_{-}$, that this reduction space is symplectomorphic to the phase space for AKS systems. This means, in particular, that the solutions of AKS systems can be 
obtained from the solution curves of a suitable Hamiltonian system on $\Psi^{-1}\left(g_{-}, \eta_{-}\right)$by taking the quotient by the $G_{+}$-action: The Hamiltonian vector field described using the Dirac brackets projects onto the Hamiltonian vector field driving the AKS flow.

To put in practice these constructions, we apply them on two examples: in the first one, we choose $G_{-}$as a representation space $V$ of $G_{+}$, regarded as the Abelian group underlying the vector space $V$, in such a way that $G_{+}$and $V$ are merged in a bigger Lie group by the semidirect product structure; in the second example, we consider the group $S L(2, \mathbb{C})$ which factorizes as $S U(2) \times B$, where $B$ is the solvable group of $2 \times 2$ complex upper triangular matrices with real positive diagonal elements and determinant 1 . We work out a collective Hamiltonian model on a generic fiber of $\Psi: S L(2, \mathbb{C}) \times \mathfrak{s l}_{2}^{*} \longrightarrow B \times \mathfrak{b}^{*}$, and construct the corresponding Lagrangian version. The study of this example is motivated by the connection between integrable systems and Poisson-Lie T-duality described in Ref. 5. The results of the current work generalize the models studied there and may be useful to enlarge the scheme for this duality.

This work is organized as follows: in Sec. II, we carry out a brief review of the Dirac procedure addressed to deal with level set of submersive maps from the phase space to another manifolds; in Sec. III we define, by means of the Dirac procedure developed in Sec. II, a class of phase spaces in the cotangent bundle of a factorizable Lie group, working out many issues as fundamental brackets, symmetries, and Hamilton equations; in Sec. IV, the connection with integrable models and factorization problem is studied from the Dirac procedure and symplectic reduction points of view. Finally, in Sec. V we present the examples, and in Sec. VI some conclusions are summarized.

\section{A BRIEF REVIEW OF DIRAC PROCEDURE}

Let $(\mathcal{M}, \omega)$ be a symplectic manifold and $\left(\mathcal{N}, \imath^{*} \omega\right)$ a symplectic submanifold with the immersion $\imath: \mathcal{N} \hookrightarrow \mathcal{M}$.

Lemma: The tangent space at a point $\iota(n) \in \mathcal{M}$ can be decomposed in a direct sum as

$$
T_{l(n)} \mathcal{M}=\iota_{*}\left(T_{n} \mathcal{N}\right) \oplus\left[l_{*}\left(T_{n} \mathcal{N}\right)\right]^{\omega \perp} .
$$

Proof: Here, $[V]^{\omega \perp}$ means the symplectic orthogonal of $V$ by the 2 -form $\omega$. This result stems from the nondegeneracy of $\omega$.

Lemma: Let $F \in \mathbb{C}^{\infty}(\mathcal{M}), V_{F} \in \mathfrak{X}(\mathcal{M})$ its Hamiltonian vector field and $\left.\pi_{\mathcal{N}}\right|_{l(n)}: T_{l(n)} \mathcal{M} \longrightarrow$ $l_{*}\left(T_{n} \mathcal{N}\right)$ the projection. Then

$$
\left.l_{*} V_{l^{*} F}\right|_{l(n)}=\left.\pi_{\mathcal{N}}\left(V_{F}\right)\right|_{l(n)}
$$

The Dirac procedure arises from considering a submanifold defined as the level surfaces of some submersive map $\Psi$ from $\mathcal{M}$ into another manifold $\mathcal{P}$. So, for some regular value $p_{\circ} \in \mathcal{P}$, we get a submanifold $\mathcal{N}$ defined as

$$
l(\mathcal{N})=\Psi^{-1}\left(p_{\circ}\right)
$$

The following theorem is in truly realm of Dirac's idea.

Theorem: Let $\Psi: \mathcal{M} \rightarrow \mathcal{P}$ a surjective map, and $p_{\circ} \in \mathcal{P}$ a regular value of $\Psi$. Then, the cotangent space at a point $\iota(n) \in \mathcal{M}$ is decomposed as

$$
T_{l(n)}^{*} \mathcal{M}=\omega\left(T_{n} \mathcal{N}\right) \oplus \Psi^{*} T_{p_{\circ}}^{*} \mathcal{P}
$$

Proof: For $n \in \Psi^{-1}\left(p_{\circ}\right)$, we have the exact short sequence

$$
0 \longrightarrow T_{n} \mathcal{N} \stackrel{l_{*}}{\longrightarrow} T_{l(n)} \mathcal{M} \stackrel{\Psi_{*(n)}}{\longrightarrow} T_{p_{\circ}} \mathcal{P} \longrightarrow 0
$$


and by duality

$$
0 \longrightarrow T_{p_{0}}^{*} \mathcal{P} \stackrel{\Psi_{l(n)}^{*}}{\longrightarrow} T_{l(n)}^{*} \mathcal{M} \stackrel{\iota^{*}}{\longrightarrow} T_{n}^{*} \mathcal{N} \longrightarrow 0
$$

so that

$$
T_{n}^{*} \mathcal{N}=\iota^{*}\left[\frac{T_{l(n)}^{*} \mathcal{M}}{\Psi_{l(n)}^{*}\left(T_{p_{\circ}}^{*} \mathcal{P}\right)}\right]
$$

from where we conclude that

$$
\left(l_{*}\left(T_{n} \mathcal{N}\right)\right)^{\circ}=\Psi_{l(n)}^{*}\left(T_{p_{\circ}}^{*} \mathcal{P}\right)
$$

Now, since $\omega \mid l(\mathcal{N})$ is nondegenerate, $\omega\left(l_{*}\left(T_{n} \mathcal{N}\right)\right)$ is complementary to $\left(l_{*}\left(T_{n} \mathcal{N}\right)\right)^{\circ}$ in $T_{l(n)}^{*} \mathcal{M}$, then

$$
T_{l(n)}^{*} \mathcal{M}=\omega\left(l_{*}\left(T_{n} \mathcal{N}\right)\right) \oplus \Psi_{l(n)}^{*}\left(T_{p_{\circ}}^{*} \mathcal{P}\right)
$$

Proposition: The induced bijection $\omega: T \mathcal{M} \longrightarrow T^{*} \mathcal{M}$ provides the isomorphism

$$
\Psi_{l(n)}^{*}\left(T_{p_{\circ}}^{*} \mathcal{P}\right) \stackrel{\omega}{\simeq}\left[l_{*}\left(T_{n} \mathcal{N}\right)\right]^{\omega \perp}
$$

for all $n \in \mathcal{N}$.

Proof: First, observe that $\left(\Psi^{*} \alpha\right)_{l(n)} \in\left[\Psi^{*}\left(T_{p_{\circ}}^{*} \mathcal{P}\right)\right]_{l(n)}, \alpha \in T_{p_{\circ}}^{*} \mathcal{P}$, the bijection $\omega$ assigns a $V_{\alpha} \in T_{l(n)} \mathcal{M}$, such that $i_{V_{\alpha}} \omega=d \Psi^{*} \alpha$. Then, $\forall W \in i_{*}\left(T_{n} \mathcal{N}\right)=\operatorname{ker} \Psi_{* l(n)}$ we have

$$
\left\langle\omega, V_{\alpha} \otimes W\right\rangle=\left\langle d \Psi^{*} \alpha, W\right\rangle=\left\langle d \alpha, \Psi_{*} W\right\rangle=0,
$$

then $V_{\alpha} \in\left[\imath_{*}\left(T_{n} \mathcal{N}\right)\right]^{\omega \perp}$.

Reciprocally, $\quad \forall W \in\left[l_{*}\left(T_{n} \mathcal{N}\right)\right]^{\omega \perp}, \exists \alpha_{W} T_{\imath(n)}^{*} \mathcal{M} / i_{W} \omega=\alpha_{W} . \quad$ Moreover, $\quad \forall V \in l_{*}\left(T_{n} \mathcal{N}\right)$ $=\operatorname{ker} \Psi_{* l(n)}$,

$$
\left\langle\alpha_{W}, V\right\rangle=\langle\omega, W \otimes V\rangle=0,
$$

then $\alpha_{W} \in\left(l_{*}\left(T_{n} \mathcal{N}\right)\right)^{o}=\Psi_{l(n)}^{*}\left(T_{p_{\circ}}^{*} \mathcal{P}\right)$.

Before going on, it is worth to distinguish the two following situations which applies into the most important physical systems:

1. First class $\Longleftrightarrow\left[\imath_{*}\left(T_{n} \mathcal{N}\right)\right]^{\omega \perp} \subset l_{*}\left(T_{n} \mathcal{N}\right) \Longleftrightarrow \mathcal{N}$ is a coisotropic submanifold.

2. Second class $\Longleftrightarrow\left[\imath_{*}\left(T_{n} \mathcal{N}\right)\right]^{\omega \perp} \cap \iota_{*}\left(T_{n} \mathcal{N}\right)=\{0\}$.

From now on, we shall be concerned with the second class case.

Theorem: $\mathcal{N}=\Psi^{-1}\left(p_{\circ}\right)$ is symplectic if and only if

$$
\left.\omega\left(\operatorname{ker} \Psi_{* l(n)}\right) \cap \Psi^{*}\left(T_{p_{\circ}}^{*} \mathcal{P}\right)\right|_{l(n)}=\{0\} .
$$

Proof: Since $p_{\circ}$ is a regular value of $\Psi$, and $\mathcal{N}$ is a level surface of $\Psi$, $\operatorname{ker} \Psi_{* l}(n)$ $=l_{*}\left(T_{n} \mathcal{N}\right)$. Then, because $\omega: T_{n} \mathcal{N} \longrightarrow T_{n}^{*} \mathcal{N}$ is a bijection, there is a one-to-one correspondence between tangent vectors to $\mathcal{N}$ and linear forms. So that seeking for null vectors of $\iota^{*} \omega$ in $T_{n} \mathcal{N}$ is equivalent to look for $\alpha \in \omega\left(\operatorname{ker} \Psi_{* l(n)}\right)$ vanishing on $T_{n} \mathcal{N}$, that is $\alpha \in \omega\left(\operatorname{ker} \Psi_{* l(n)}\right) \cap\left(l_{*}\left(T_{n} \mathcal{N}\right)\right)^{\mathrm{o}}$. Regularity of $\iota^{*} \omega$ implies no nontrivial such an $\alpha$ does exist, so $\left.\omega\left(\operatorname{ker} \Psi_{* l(n)}\right) \cap \Psi^{*}\left(T_{p_{\circ}}^{*} \mathcal{P}\right)\right|_{l(n)}$ $=\{0\}$.

Corollary: $\Psi$ is a second class constraint if and only if

$$
\left.\omega\left(\operatorname{ker} \Psi_{* l(n)}\right) \cap \Psi^{*}\left(T_{p_{\circ}}^{*} \mathcal{P}\right)\right|_{l(n)}=\{0\} .
$$


Proof: $\Psi$ is a second class if and only if $\left[l_{*}\left(T_{n} \mathcal{N}\right)\right]^{\omega \perp} \cap l_{*}\left(T_{n} \mathcal{N}\right)=\{0\}$, that is equivalent to $\left(l_{*}\left(T_{n} \mathcal{N}\right)\right)^{\circ} \cap \operatorname{ker} \Psi_{* l(n)}=\{0\}$. And it happens if and only if $\left.\omega\left(\operatorname{ker} \Psi_{* l(n)}\right) \cap \Psi^{*}\left(T_{p_{\circ}}^{*} \mathcal{P}\right)\right|_{l(n)}$ $=\{0\}$.

Let us now introduce a suitable set of functions $\left\{f_{1}, \cdots, f_{r}\right\} \subset \mathbb{C}^{\infty}(\mathcal{P}), r=\operatorname{dim} \mathcal{P}$, such that $\left\{\left.d f_{1}\right|_{p_{\circ}}, \cdots,\left.d f_{r}\right|_{p_{0}}\right\}$ is a basis of $T_{p_{o}}^{*} \mathcal{P}$. Then, $\left\{f_{1}, \cdots, f_{r}\right\}$ is a local coordinate system for $\mathcal{P}$. Let us consider then the set of local Hamiltonian vector fields associated with the pullback of these functions

$$
\Psi^{*} f_{j} \longrightarrow V_{\Psi^{*} f_{j}} / l_{{\Psi^{*} f_{j}}_{j}} \omega=d\left(\Psi^{*} f_{j}\right)
$$

Proposition: Let $\Psi$ be a second class constraint, then the set $\left\{V_{\Psi^{*} f_{j}}\right\}_{i=1}^{r}$ is a basis for $\left[l_{*}\left(T_{n} \mathcal{N}\right)\right]^{\omega \perp}$.

Proof: Let be $W \in \imath_{*}\left(T_{n} \mathcal{N}\right)=\operatorname{ker} \Psi_{* l(n)}$, then

$$
\left\langle\omega, V_{\Psi^{*} f_{j}} \otimes W\right\rangle_{\iota_{(n)}}=\left\langle d\left(\Psi^{*} f_{j}\right), W\right\rangle_{\iota(n)}=\left\langle d f_{j}, \Psi_{*} W\right\rangle_{\Psi(n)}=0
$$

from where we conclude that each $V_{\Psi^{*} f_{j}} \in\left[l_{*}\left(T_{n} \mathcal{N}\right)\right]^{\omega \perp}$.

One may see that the functions $\Psi^{*} f_{1}, \cdots, \Psi^{*} f_{r}$ are constant on $\mathcal{N}=\Psi^{-1}\left(p_{\circ}\right)$. Also, we know that $\left\{\left.d f_{1}\right|_{p_{\circ}}, \cdots,\left.d f_{r}\right|_{p_{\circ}}\right\}$ is a linearly independent set, then so is $\left\{\left.d \Psi^{*} f_{1}\right|_{l(n)}, \cdots,\left.d \Psi^{*} f_{r}\right|_{l(n)}\right\}$ because $\Psi$ is a surjective map and $\Psi^{*}$ is an injective one. Then, the set $\left\{V_{\Psi^{*} f_{j}}\right\}_{i=1}^{r}$ is linearly independent too.

From Theorem (3) we know that $T_{l(n)}^{*} \mathcal{M}=\omega\left(l_{*}\left(T_{n} \mathcal{N}\right)\right) \oplus \Psi_{l(n)}^{*}\left(T_{p_{\circ}}^{*} \mathcal{P}\right)$, and because $\omega$ is nondegenerate on $l_{*}\left(T_{n} \mathcal{N}\right)$, we can apply $\omega^{-1}$ on this direct sum to map it to the tangent space,

$$
T_{l(n)} \mathcal{M}=\iota_{*}\left(T_{n} \mathcal{N}\right) \oplus \omega^{-1}\left(\Psi_{l(n)}^{*}\left(T_{p_{\circ}}^{*} \mathcal{P}\right)\right),
$$

and this decomposition is symplectically orthogonal. This shows that

$$
\omega^{-1}\left(\Psi_{\imath(n)}^{*}\left(T_{p_{0}}^{*} \mathcal{P}\right)\right)=\left[\iota_{*}\left(T_{n} \mathcal{N}\right)\right]^{\omega \perp} .
$$

This finishes the proof.

Corollary: The set,

$$
\left\{\left.d \Psi^{*} f_{1}\right|_{l(n)}, \cdots,\left.d \Psi^{*} f_{r}\right|_{l(n)}\right\} \subset T_{l(n)}^{*} \mathcal{M},
$$

is a basis of $\left[l_{*}\left(T_{n} \mathcal{N}\right)\right]^{\circ}=\Psi_{l(n)}^{*}\left(T_{p_{\circ}}^{*} \mathcal{P}\right)$.

Then, from the last four assertions, we conclude that in the second class case any vector $V \in T_{l(n)} \mathcal{M}$ can be written as

$$
V_{l(n)}=\left(l_{*} v\right)_{l(n)}+\sum_{i=1}^{r} a^{i}(n) V_{\Psi^{*} f_{i}},
$$

where $v \in T_{n} \mathcal{N}$. Let us introduce the Dirac matrix

$$
C_{j k}(l(n))=\left\langle d \Psi^{*} f_{j}, V_{\Psi^{*} f_{k}}\right\rangle_{l(n)}=\left\{\Psi^{*} f_{j}, \Psi^{*} f_{k}\right\}(l(n)) .
$$

Observe that, in the case of $\Psi$ being second class, $\left.\omega\left(l_{*}\left(T_{n} \mathcal{N}\right)\right) \cap \Psi^{*}\left(T_{p_{\circ}}^{*} \mathcal{P}\right)\right|_{l(n)}=\{0\}$, there are no Hamiltonian forms associated with vector of $l_{*}\left(T_{n} \mathcal{N}\right)$ contained in $\left.\Psi^{*}\left(T_{p_{\circ}}^{*} \mathcal{P}\right)\right|_{l_{(n)}}$. Then, in this case the vector space $\Psi_{l(n)}^{*}\left(T_{p_{\circ}}^{*} \mathcal{P}\right)=\left[l_{*}\left(T_{n} \mathcal{N}\right)\right]^{\circ}$ is the dual of $\left[l_{*}\left(T_{n} \mathcal{N}\right)\right]^{\omega \perp}$,

$$
\Psi_{l(n)}^{*}\left(T_{p_{\circ}}^{*} \mathcal{P}\right)=\left(\left[l_{*}\left(T_{n} \mathcal{N}\right)\right]^{\omega \perp}\right)^{*},
$$

so that the matrix obtained from the contraction of both the basis $\left\{\left.d \Psi^{*} f_{j}\right|_{l(n)}\right\}$ and $\left\{V_{\Psi^{*} f_{j}}\right\}_{i=1}^{r}$, namely, $C_{j k}(l(n))$ is an invertible one. 
Contracting the vector $V_{l(n)}$ with $d \Psi^{*} f_{j}$, and because $\left\langle d \Psi^{*} f_{k}, l_{*} v\right\rangle_{l(n)}=\left\langle d f_{k}, \Psi_{*} l_{*} v\right\rangle_{p_{\circ}}=0$ (remember that $\left.l_{*}\left(T_{n} \mathcal{N}\right)=\operatorname{ker} \Psi_{* l(n)}\right)$, we get

$$
a^{j}(n)=\sum_{k=1}^{r} C^{j k}(l(n))\left\langle d \Psi^{*} f_{k}, V\right\rangle_{\iota(n)} .
$$

Writing the vector $V, W$ in terms of the expression (6), including the coefficients given in the last equation, we get from the contraction $\langle\omega, V \otimes W\rangle_{l(n)}$ the following relation:

$$
\left\langle l^{*} \omega, v \otimes w\right\rangle_{n}=\langle\omega, V \otimes W\rangle_{l(n)}+\left\langle\sum_{k, l=1}^{r} C^{l k}(l(n)) \Psi^{*}\left(d f_{l} \wedge d f_{k}\right), V \otimes W\right\rangle_{l(n)} .
$$

Lemma: Let be $\alpha \in \Omega^{1}(\mathcal{M})$ and let be $V_{\alpha} \in \mathfrak{X}(\mathcal{M})$, the associated Hamiltonian vector field. Let the surjection $\Psi: \mathcal{M} \longrightarrow \mathcal{P}$ be a second class constraint, and $\left.\pi_{\mathcal{N}}\right|_{n}: T_{l(n)} \mathcal{M} \longrightarrow T_{n} \mathcal{N}$ the projection on the first factor in the direct sum in (6). Then

$$
l_{*} v_{l^{*} \alpha}=\pi_{\mathcal{N}} V_{\alpha} .
$$

So, let us now specialize the expression of $\left\langle l^{*} \omega, v \otimes w\right\rangle_{n}$ given above to the Hamiltonian vector fields, such as in the last Lemma, and defining as usual

$$
\{f, g\}^{\mathcal{N}}(n)=\left\langle\imath^{*} \omega, v_{f} \otimes v_{g}\right\rangle_{n},
$$

$\forall f, g \in \mathbb{C}^{\infty}(\mathcal{N})$, we obtain the celebrated Dirac formula relating the Poisson bracket on $\mathcal{N}$ with the one defined on $\mathcal{M}$,

$$
\begin{aligned}
\left\{l^{*} F, l^{*} H\right\}^{\mathcal{N}}(n)= & \{F, H\}^{\mathcal{M}}(l(n)) \\
& -\sum_{l, k=1}^{r}\left\{F, \Psi^{*} f_{l}\right\}^{\mathcal{M}}{ }_{l}(n) C^{l k}(l(n))\left\{\Psi^{*} f_{k}, H\right\}^{\mathcal{M}}(l(n)) .
\end{aligned}
$$

\section{SYMPLECTIC SUBMANIFOLDS IN COTANGENT BUNDLE OF DOUBLE LIE GROUP AS CONSTRAINED SYSTEM}

In this section, we shall consider tangent and cotangent bundles of Lie groups trivialized by left translation, a procedure equivalent to take global body coordinates.

Given three Lie groups $\left(G, G_{+}, G_{-}\right)$, where $G_{+}$and $G_{-}$are both closed Lie subgroups of $G$, we say they form a double Lie group if there exist a diffeomorphism $\alpha: G_{+} \times G_{-} \longrightarrow G$ defined as $\left(g_{+}, g_{-}\right) \rightarrow g_{+} g_{-} .{ }^{7}$ In this case, the Lie algebras $\left(\mathfrak{g}, \mathfrak{g}_{+}, \mathfrak{g}_{-}\right)$form a double Lie algebra if $\mathfrak{g}_{+}$and $\mathfrak{g}_{-}$are Lie subalgebras of $\mathfrak{g}$, and $\mathfrak{g}=\mathfrak{g}_{+} \oplus \mathfrak{g}_{-}$as vector spaces. By duality, this decomposition also gives rise to the factorization of $\mathfrak{g}^{*}=\mathfrak{g}_{-}^{\circ} \oplus \mathfrak{g}_{+}^{\circ}$, where

$$
\mathfrak{g}_{ \pm}^{\circ}=\left\{\eta \in \mathfrak{g}^{*} /\left\langle\eta, X_{ \pm}\right\rangle=0, \forall X_{ \pm} \in \mathfrak{g}_{ \pm}^{\circ}\right\}
$$

that allows to make the identifications $\mathfrak{g}_{ \pm}^{*} \cong \mathfrak{g}_{\mp}^{\circ}$. In particular, if $\mathfrak{g}$ is supplied with an $A d^{G}$-invariant nondegenerate symmetric bilinear form turning $\mathfrak{g}_{+}^{\circ}, \mathfrak{g}_{-}^{\circ}$ into isotropic subspaces, the previous identifications imply $\mathfrak{g}_{ \pm}^{*} \cong \mathfrak{g}_{\mp}$. Let us denote the corresponding projectors $\Pi_{G_{ \pm}}: G \longrightarrow$ $G_{ \pm}, \Pi_{\mathfrak{g}_{ \pm}^{\circ}}: \mathfrak{g} \longrightarrow \mathfrak{g}_{ \pm}^{\circ}$, and $\Pi_{\mathfrak{g}_{ \pm}^{*}}: \mathfrak{g}^{*} \longrightarrow \mathfrak{g}_{ \pm}^{*}$. We also denote $\eta_{ \pm}=\Pi_{\mathfrak{g}_{ \pm}^{*}} \eta$.

Writing out every element $g \in G$ as $g=g_{+} g_{-}$, with $g_{+} \in G_{+}$and $g_{-} \in G_{-}$, the product $g_{-} g_{+}$ in $G$ can be expressed as $g_{-} g_{+}=g_{+}^{g_{-}} g_{-}^{g_{+}}$, with $g_{+}^{g_{-}} \in G_{+}$and $g_{-}^{g_{+}} \in G_{-}{ }^{7,13}$ The dressing action of $G_{-}$on $G_{+}$is defined to be

$$
\operatorname{Dr}: G_{-} \times G_{+} \longrightarrow G_{+} \quad / \quad \operatorname{Dr}\left(h_{-}, g_{+}\right):=\Pi_{G_{+}} h_{-} g_{+}=g_{+}^{h_{-}} .
$$

The infinitesimal generator of this action at the point $g_{+} \in G_{+}$is, for $X_{-} \in \mathfrak{g}_{-}$,

$$
X_{-} \longrightarrow g_{+}^{X_{-}}=-\left.\frac{d}{d t} \operatorname{Dr}\left(e^{t X_{-}}, g_{+}\right)\right|_{t=0}
$$


such that, for $X_{-}, Y_{-} \in \mathfrak{g}_{-}$, we have $\left[g_{+}^{X_{-}}, g_{+}^{Y_{-}}\right]=-g_{+}^{\left[X_{-}, Y_{-}\right]_{\mathfrak{g}_{-}}}$. It satisfies the relation

$$
A d_{g_{+}^{-1}}^{G} X_{-}=g_{+}^{-1} g_{+}^{X_{-}}+A d_{g_{+}}^{*} X_{-},
$$

where $A d_{g_{+}^{-1}}^{G} X_{-} \in \mathfrak{g}$ is the adjoint action of $G$, and $A d_{g_{+}}^{*} X_{-} \in \mathfrak{g}_{-}$is the coadjoint action of $G_{+}$on the dual of its Lie algebra $\mathfrak{g}_{+}^{*} \cong \mathfrak{g}_{-}^{\circ}$. Then, we may write $g_{+}^{X_{-}}=g_{+} \Pi_{\mathfrak{g}_{+}} A d_{g_{+}^{-1}}^{G} X_{-}$.

Let us introduce the fibrations

$$
\begin{gathered}
\Psi: G \times \mathfrak{g}^{*} \longrightarrow G_{-} \times \mathfrak{g}_{-}^{*} \\
(g, \eta) \longmapsto\left(\Pi_{G_{-}}(g), \Pi_{\mathfrak{g}_{-}^{*}}(\eta)\right), \\
\Upsilon: G \times \mathfrak{g}^{*} \longrightarrow G_{+} \times \mathfrak{g}_{+}^{*} \\
(g, \eta) \longmapsto\left(\Pi_{G_{+}}(g), \Pi_{\mathfrak{g}_{+}^{*}}(\eta)\right),
\end{gathered}
$$

with fibers

$$
\begin{aligned}
& \mathcal{N}\left(g_{-}, \eta_{-}\right)=\left\{(g, \eta) \in G \times \mathfrak{g}^{*} / \Psi(g, \eta)=\left(g_{-}, \eta_{-}\right)\right\}, \\
& \mathcal{M}\left(g_{+}, \eta_{+}\right)=\left\{(g, \eta) \in G \times \mathfrak{g}^{*} / \Upsilon(g, \eta)=\left(g_{+}, \eta_{+}\right)\right\} .
\end{aligned}
$$

Observe that the corresponding fibers on $(e, 0) \in G_{-} \times \mathfrak{g}_{-}^{*}$ reduce to the trivialized cotangent bundles of the factors $G_{+}, G_{-}$, namely, $\mathcal{N}(e, 0)=G_{+} \times \mathfrak{g}_{+}^{*} \cong T^{*} G_{+}$and $\mathcal{M}(e, 0)=G_{-} \times \mathfrak{g}_{-}^{*} \cong T^{*} G_{-}$.

The differential of the maps $\Psi$ and $\Upsilon$ involves the following digression: a vector $v \in T_{g} G$ with $g=g_{+} g_{-}$can be written as

$$
v=v_{+} g_{-}+g_{+} v_{-}
$$

that in terms of left translated vectors on $G_{+} \times \mathfrak{g}_{+}^{\circ}$ and $G_{-} \times \mathfrak{g}_{-}^{\circ}$ reads

$$
\dot{g}=g_{+}\left(g_{+}^{-1} v_{+}\right) g_{-}+g_{+} g_{-}\left(g_{-}^{-1} v_{-}\right) .
$$

Hence, the relation with the left trivialization of $T G \cong G \times \mathfrak{g}$ arises from

$$
g^{-1} v=A d_{g_{-}^{-1}}^{G}\left(g_{+}^{-1} v_{+}\right)+\left(g_{-}^{-1} v_{-}\right)
$$

from where we define

$$
\begin{aligned}
& X_{+}=\Pi_{\mathfrak{g}_{+}^{\circ}} g^{-1} v=A d_{g_{-}}^{*}\left(g_{+}^{-1} v_{+}\right), \\
& X_{-}=\Pi_{\mathfrak{g}_{-}^{\circ}} g^{-1} v=\left(g_{-}^{-1}\right)^{g_{+}^{-1} v_{+}} g_{-}+g_{-}^{-1} v_{-}
\end{aligned}
$$

that are equivalent to

$$
\left\{\begin{array}{l}
g_{+}^{-1} \dot{g}_{+}=A d_{g_{-}^{-1}}^{*} X_{+} \\
g_{-}^{-1} \dot{g}_{-}=X_{-}+g_{-}^{-1} g_{-}^{X_{+}} .
\end{array}\right.
$$

We shall use these results in Secs. IV and V, where $\mathcal{N}\left(g_{-}, \eta_{-}\right)$and $\mathcal{M}\left(g_{+}, \eta_{+}\right)$shall be regarded as phase spaces.

In fact, since the cotangent bundle $T^{*} G \cong G \times \mathfrak{g}^{*}$ is a symplectic manifold with the canonical 2 -form $\omega_{\circ}$, which has associated the nondegenerate Poisson bracket,

$$
\{\mathcal{F}, \mathcal{G}\}(g, \eta)=\langle g \mathbf{d} \mathcal{F}, \delta \mathcal{G}\rangle_{(g, \eta)}-\langle g \mathbf{d} \mathcal{G}, \delta \mathcal{F}\rangle_{(g, \eta)}-\left\langle\eta,\left[\left.d \mathcal{F}\right|_{(g, \eta)},\left.d \mathcal{G}\right|_{(g, \eta)}\right]\right\rangle,
$$

where we wrote $\left.d \mathcal{F}\right|_{(g, \eta)}=\left.(\mathbf{d} \mathcal{F}, \delta \mathcal{G})\right|_{(g, \eta)} \in T_{g}^{*} G \oplus \mathfrak{g}^{*}$, we shall study how to apply the Dirac method to supply the submanifolds $\mathcal{N}\left(g_{-}, \eta_{-}\right)$and $\mathcal{M}\left(g_{+}, \eta_{+}\right)$with a Poisson-Dirac structure. 


\section{A. The phase spaces $\mathcal{N}\left(g_{-}, \eta_{-}\right)$}

Let us consider the restriction of the canonical Poisson bracket of $T^{*} G \cong G \times \mathfrak{g}^{*}$ to the fibers $\mathcal{N}\left(g_{-}, \eta_{-}\right):=\Psi^{-1}\left(g_{-}, \eta_{-}\right)$. Then the differential map $\Psi_{*}: T\left(G \times \mathfrak{g}^{*}\right) \longrightarrow T\left(G_{-} \times \mathfrak{g}_{-}^{*}\right)$ is

$$
\left.\Psi_{*}(g X, \xi)\right|_{(g, \eta)}=\left.\frac{d}{d t} \Pi_{G_{-}}\left(g e^{t X}\right)\right|_{t=0}=\left(g_{-}^{X_{+}}+g_{-} X_{-}, \xi_{-}\right)_{\left(g_{-}, \eta_{-}\right)} .
$$

Therefore, the kernel of $\Psi_{*}$, that coincides with $T \mathcal{N}\left(g_{-}, \eta_{-}\right)$, is

$$
\left.\operatorname{ker} \Psi_{*}\right|_{(g, \eta)}=\left\{\left(g_{+}\left(A d_{g_{-}^{-1}}^{*} X_{+}\right) g_{-}, \xi_{+}\right) /\left(X_{+}, \xi_{+}\right) \in \mathfrak{g}_{+}^{\circ} \oplus \mathfrak{g}_{+}^{*}\right\} .
$$

At each point $(g, \eta) \in \mathcal{N}\left(g_{-}, \eta_{-}\right)$, the intersection of $T_{(g, \eta)} \mathcal{N}\left(g_{-}, \eta_{-}\right)$with its symplectic orthogonal is $\{0\}$, so we have the following result.

Proposition: $\left(\mathcal{N}\left(g_{-}, \eta_{-}\right), \tilde{\omega}_{\circ}\right)$, where $\tilde{\omega}_{\circ}$ is the restriction to $\mathcal{N}\left(g_{-}, \eta_{-}\right)$of the canonical symplectic form $\omega_{\circ}$ on $G \times \mathfrak{g}^{*}$, is a symplectic manifold.

Proof: The restriction of the symplectic form to $T \mathcal{N}\left(g_{-}, \eta_{-}\right)$reduces to

$$
\begin{aligned}
& \left\langle\omega_{\circ},\left(-g_{+}\left(A d_{g_{-}^{-1}}^{*} X_{+}\right) g_{-}, \xi_{+}\right) \otimes\left(-g_{+}\left(A d_{g_{-}^{-1}}^{*} Y_{+}\right) g_{-}, \lambda_{+}\right)\right\rangle_{(g, \eta)} \\
& =-\left\langle\xi_{+}, Y_{+}\right\rangle+\left\langle\lambda_{+}, X_{+}\right\rangle+\left\langle A d_{g_{-}} \eta_{+},\left[A d_{g_{-}^{-1}}^{*} X_{+}, A d_{g_{-}^{-1}}^{*} Y_{+}\right]\right\rangle \\
& \left.\quad+\left\langle\eta_{-},\left(g_{-}^{-1}\right)^{\left[A d_{g_{-}^{-1}}^{*} X_{+}, A d_{g_{-}}^{*} Y_{+}\right.}\right]_{g_{-}}\right\rangle .
\end{aligned}
$$

Here, we can see that there are no null vectors of $\omega_{\circ}$ on $T \Psi^{-1}\left(g_{-}, \eta_{-}\right)=\operatorname{ker} \Psi_{*} \mid(g, \eta)$.

Corollary: $\left(T_{(g, \eta)} \mathcal{N}\left(g_{-}, \eta_{-}\right)\right)^{\omega_{\circ} \perp} \cap T_{(g, \eta)} \mathcal{N}\left(g_{-}, \eta_{-}\right)=\{0\}$, then $\mathcal{N}\left(g_{-}, \eta_{-}\right)$is a second class constraint.

\section{Dirac brackets on $\mathcal{N}\left(g_{-}, \eta_{-}\right)$}

In order to built up the Dirac brackets, we choose a basis for $T_{\left(g_{-}, \eta_{-}\right)}^{*}\left(G_{-} \times \mathfrak{g}_{-}^{*}\right) \cong \mathfrak{g}_{-}^{*} \oplus \mathfrak{g}_{-}^{\circ}$. In doing so, we introduce the basis $\left\{T_{a}\right\}_{a=1}^{n}$ of $\mathfrak{g}_{+}^{\circ} \cong \mathfrak{g}_{-}^{*}$ and the basis $\left\{T^{a}\right\}_{a=1}^{n}$ of $\mathfrak{g}_{-}^{\circ}=\mathfrak{g}_{+}^{*}$, which provide a set of linearly independent 1 -forms on $G_{-} \times \mathfrak{g}_{-}^{*}$,

$$
\begin{aligned}
& \alpha_{a}=\left(L_{g_{-}^{-1}}^{*} T_{a}, 0\right) \in T_{\left(g_{-}, \eta_{-}\right)}^{*} G_{-} \times \mathfrak{g}_{-}^{*}, \\
& \beta_{a}=\left(0, T^{a}\right) \in T_{\left(g_{-}, \eta_{-}\right)}^{*} G_{-} \times \mathfrak{g}_{-}^{*},
\end{aligned}
$$

for $a=1,2, \ldots, n$. Their pullback amount a set $\left\{\Psi^{*} \alpha_{a}\right\}_{a=1}^{n} \cup\left\{\Psi^{*} \beta^{a}\right\}_{a=1}^{n}$ of linearly independent 1 -forms on $G \times \mathfrak{g}^{*}$, whose null distribution is precisely the tangent space of $\mathcal{N}\left(g_{-}, \eta_{-}\right)$.

Hence, the Hamiltonian vector fields associated with these forms through $\omega_{\circ}$ are

$$
\begin{aligned}
& V_{\Psi^{*} \alpha_{a}}=\left(0, g_{-}^{-1} g_{-}^{T_{a}}-T_{a}\right)_{(g, \eta)}, \\
& V_{\Psi^{*} \beta^{a}}=\left(g T^{a}, a d_{T^{a}}^{\mathfrak{g} *} \eta\right) .
\end{aligned}
$$

So, we calculate the entries of the following Dirac matrix:

$$
\begin{aligned}
& C_{\Psi^{*} \alpha_{a}, \Psi^{*} \alpha_{b}}(g, \eta)=\left\langle\Psi^{*} \alpha_{a}, V_{\Psi^{*} \alpha_{b}}\right\rangle_{(g, \eta)}=0, \\
& C_{\Psi^{*} \beta^{a}, \Psi^{*} \beta^{b}}(g, \eta)=\left\langle\Psi^{*} \beta^{a}, V_{\Psi^{*} \beta^{b}}\right\rangle_{(g, \eta)}=-\left\langle\eta_{-},\left[T^{a}, T^{b}\right]\right\rangle, \\
& C_{\Psi^{*} \alpha_{a}, \Psi^{*} \beta^{b}}(g, \eta)=\left\langle\Psi^{*} \alpha_{a}, V_{\Psi^{*} \beta^{b}}\right\rangle_{(g, \eta)}=\delta_{a}^{b},
\end{aligned}
$$


that finally produces the matrix

$$
C(g, \eta)=\left[\begin{array}{cc}
0_{n \times n} & -I_{n \times n} \\
I_{n \times n} & \Omega(\eta)
\end{array}\right],
$$

where $\Omega(\eta)$ stands for the $n \times n$ matrix of entries

$$
\Omega_{a b}(\eta)=-\left\langle\eta_{-},\left[T^{a}, T^{b}\right]\right\rangle
$$

Now, we are ready to introduce the Dirac brackets: carrying these results in the expression (8) for any couple of function $\mathcal{F}, \mathcal{G} \in C^{\infty}\left(G \times \mathfrak{g}^{*}\right)$, the Dirac bracket gives the restriction of Poisson bracket on $G \times \mathfrak{g}^{*}$ to constraint submanifold $\mathcal{N}\left(g_{-}, \eta_{-}\right)$and it is defined as

$$
\begin{aligned}
\{\mathcal{F}, \mathcal{G}\}^{\mathcal{N}}(g, \eta)= & \{\mathcal{F}, \mathcal{G}\}(g, \eta)-\left\{\mathcal{F}, \alpha_{a}\right\}(g, \eta) \Omega_{a b}(\eta)\left\{\alpha_{b}, \mathcal{G}\right\}(g, \eta) \\
& +\left\{\mathcal{F}, \alpha_{a}\right\}(g, \eta)\left\{\beta^{a}, \mathcal{G}\right\}(g, \eta)-\left\{\mathcal{F}, \beta^{a}\right\}(g, \eta)\left\{\alpha_{a}, \mathcal{G}\right\}(g, \eta),
\end{aligned}
$$

that has the explicit form

$$
\begin{aligned}
\{\mathcal{F}, \mathcal{G}\}^{\mathcal{N}}(g, \eta)= & \left\langle g \mathbf{d} \mathcal{F}, A d_{g_{-}^{-1}}^{G} \Pi_{\mathfrak{g}_{+}^{\circ}} A d_{g_{-}}^{G} \delta \mathcal{G}\right\rangle-\left\langle g \mathbf{d} \mathcal{G}, A d_{g_{-}^{-1}}^{G} \Pi_{\mathfrak{g}_{+}^{\circ}} A d_{g_{-}}^{G} \delta \mathcal{F}\right\rangle \\
& -\left\langle\eta,\left[A d_{g_{-}^{-1}}^{G} \Pi_{\mathfrak{g}_{+}^{\circ}} A d_{g_{-}}^{G} \delta \mathcal{F}, A d_{g_{-}^{-1}}^{G} \Pi_{\mathfrak{g}_{+}^{\circ}} A d_{g_{-}}^{G} \delta \mathcal{G}\right]\right\rangle .
\end{aligned}
$$

Observe that, for $\mathcal{N}(e, 0)$, it turns in

$$
\{\mathcal{F}, \mathcal{G}\}^{\mathcal{N}}\left(g_{+}, \eta_{+}\right)=\left\langle g \mathbf{d} \mathcal{F}, \delta \mathcal{G}_{+}\right\rangle-\left\langle g \mathbf{d} \mathcal{G}, \delta \mathcal{F}_{+}\right\rangle-\left\langle\eta_{+},\left[\delta \mathcal{F}_{+}, \delta \mathcal{G}_{+}\right]\right\rangle,
$$

giving the usual canonical Poisson structure on $T^{*} G_{+}=G_{+} \times \mathfrak{g}_{+}^{*}$.

\section{The fundamental brackets}

Let $\mathrm{T}: G \longrightarrow G L(n, \mathbb{C})$ a representation of the group $G$, with the associated set of functions $\mathrm{T}_{i}^{j}: G \longrightarrow \mathbb{C}$, such that $\mathrm{T}=E_{i}^{j} \mathrm{~T}_{i}^{j}$, being $E_{i}^{j}, i, j=1, \ldots, n$, the elementary $n \times n$ matrices with entries $\left[E_{i}^{j}\right]_{k}^{l}=\delta_{i k} \delta^{j l}$. For $g \in G$,

$$
\mathrm{T}_{i}^{j}(g)=g_{i}^{j} \Longrightarrow \mathrm{T}(g)=\sum_{i, j=1}^{n} g_{i}^{j} E_{i}^{j} .
$$

Also, we consider the coordinates $\left\{\xi_{A}\right\}_{A=1}^{N}$ for $\mathfrak{g}^{*}$, associated with the basis $\left\{\mathbb{T}_{A}\right\}_{A=1}^{N}$ of $\mathfrak{g}$, such that

$$
\xi_{A}: \mathfrak{g}^{*} \longrightarrow \mathbb{C} / \xi_{A}=\left\langle\xi, \mathbb{T}_{A}\right\rangle
$$

The representation map T induces the map $d \mathrm{~T}: \mathfrak{g} \longrightarrow g l(n, \mathbb{C})$, such that $(d \mathrm{~T})_{e} X=\sum_{i, j=1}^{n} X_{i}^{j} E_{i}^{j}$.

The corresponding fundamental Poisson bracket on $G \times \mathfrak{g}^{*}$, associated with the canonical Poisson bracket, are

$$
\begin{aligned}
\left\{\mathrm{T}_{i}^{j}, \mathrm{~T}_{k}^{l}\right\}(g, \xi) & =0, \\
\left\{\xi_{A}, \mathrm{~T}_{i}^{j}\right\}(g, \xi) & =-\sum_{k=1}^{n} g_{i}^{k}\left[T_{A}\right]_{k}^{j} .
\end{aligned}
$$

Let us now calculate the Dirac brackets for these functions. First observe that

$$
\delta \mathrm{T}_{i}^{j}=0, \mathbf{d} \xi_{A}=0,
$$

hence, carrying these differential into the Dirac bracket (20), we get the fundamental brackets on $\mathcal{N}\left(g_{-}, \eta_{-}\right)$. The differential $\delta \xi_{A}$ coincides with the generator $T_{A}$ of the Lie algebra $\mathfrak{g}$, being $T_{A} \in\left\{T_{a}, T^{a}\right\}_{a=1}^{n}$. These relations can be written in terms of the coordinates for $\eta_{+}=\left\langle\eta_{+}, T_{a}\right| \mathbf{t}_{a}$ $=\xi_{a}(\eta) \mathbf{t}_{a}$ and $\eta_{-}=\left\langle\eta_{-}, T^{a}\right\rangle \mathbf{t}^{a}=\xi^{a}(\eta) \mathbf{t}^{a}$. 
The fundamental Dirac brackets on the submanifold $\Psi^{-1}\left(g_{-}, \eta_{-}\right) \subset G \times \mathfrak{g}^{*}$ are

$$
\begin{aligned}
& \left\{\mathrm{T}_{i}^{j}, \mathrm{~T}_{k}^{l}\right\}^{D}(g, \eta)=0, \\
& \left\{\xi_{a}, \mathrm{~T}_{i}^{j}\right\}^{D}(g, \eta)=\left\langle g \mathbf{d} T_{i}^{j}, g_{-}^{-1} g_{-}^{T_{a}}-T_{a}\right\rangle, \\
& \left\{\xi^{a}, \mathrm{~T}_{i}^{j}\right\}^{D}(g, \eta)=0, \\
& \left\{\xi_{a}, \xi_{b}\right\}^{D}(g, \eta)=-\left\langle\eta,\left[T_{a}-g_{-}^{-1} g_{-}^{T_{a}}, T_{b}-g_{-}^{-1} g_{-}^{T_{b}}\right]\right\rangle, \\
& \left\{\xi_{a}, \xi^{b}\right\}^{D}(g, \eta)=0, \\
& \left\{\xi^{a}, \xi^{b}\right\}^{D}(g, \eta)=0 .
\end{aligned}
$$

Since,

$$
\left\langle g \mathbf{d} T_{i}^{j}, g_{-}^{-1} g_{-}^{T_{a}}-T_{a}\right\rangle=\mathrm{T}_{i}^{k}\left(g_{+}\right)\left[g_{-}^{T_{a}}\right]_{k}^{j}-\mathrm{T}_{i}^{k}(g)\left[T_{a}\right]_{k}^{j}
$$

and from the definition of the Lie bracket in $\mathfrak{g}=\mathfrak{g}_{+}^{\circ} \oplus \mathfrak{g}_{-}^{\circ}$,

$$
\begin{aligned}
{\left[T_{a}-g_{-}^{-1} g_{-}^{T_{a}}, T_{b}-g_{-}^{-1} g_{-}^{T_{b}}\right]=} & {\left[T_{a}, T_{b}\right]+a d_{g_{-}^{-1} g_{-}^{T_{a}}}^{*} T_{b}-a d_{g_{-}^{-1} g_{-}^{T_{b}}}^{*} T_{a} } \\
& +\left[g_{-}^{-1} g_{-}^{T_{a}}, g_{-}^{-1} g_{-}^{T_{b}}\right]+a d_{T_{a}}^{*} g_{-}^{-1} g_{-}^{T_{b}}-a d_{T_{b}}^{*} g_{-}^{-1} g_{-}^{T_{a}},
\end{aligned}
$$

the fundamental Dirac brackets can be written as

$$
\begin{aligned}
& \left\{\mathrm{T}_{i}^{j}, \mathrm{~T}_{k}^{l}\right\}^{D}(g, \eta)=0, \\
& \left\{\xi_{a}, \mathrm{~T}_{i}^{j}\right\}^{D}(g, \eta)=\mathrm{T}_{i}^{k}\left(g_{+}\right)\left[g_{-}^{T_{a}}\right]_{k}^{j}-\mathrm{T}_{i}^{k}(g)\left[T_{a}\right]_{k}^{j}, \\
& \left\{\xi^{a}, \mathrm{~T}_{i}^{j}\right\}^{D}(g, \eta)=0, \\
& \left\{\xi_{a}, \xi_{b}\right\}^{D}(g, \eta)=-f_{a b}^{c} \xi_{c}(\eta)+m_{a b}^{c}\left(g_{-}\right) \xi_{c}(\eta)+n_{a b}^{c}\left(g_{-}\right) \xi^{c}(\eta), \\
& \left\{\xi_{a}, \xi^{b}\right\}^{D}(g, \eta)=0, \\
& \left\{\xi^{a}, \xi^{b}\right\}^{D}(g, \eta)=0,
\end{aligned}
$$

where

$$
\begin{aligned}
& m_{a b}^{c}\left(g_{-}\right)=\left\langle\left[g_{-}^{-1} g_{-}^{T_{b}}, T^{c}\right], T_{a}\right\rangle-\left\langle\left[g_{-}^{-1} g_{-}^{T_{a}}, T^{c}\right], T_{b}\right\rangle, \\
& n_{a b}^{c}\left(g_{-}\right)=\left\langle\left[T_{b}, T_{c}\right], g_{-}^{-1} g_{-}^{T_{a}}\right\rangle-\left\langle\left[T_{a}, T_{c}\right], g_{-}^{-1} g_{-}^{T_{b}}\right\rangle-\left\langle T_{c},\left[g_{-}^{-1} g_{-}^{T_{a}}, g_{-}^{-1} g_{-}^{T_{b}}\right]\right\rangle,
\end{aligned}
$$

are constant coefficients on each submanifold $\mathcal{N}\left(g_{-}, \eta_{-}\right)$and, in particular, they vanish for $g_{-}=e$, $m_{a b}^{c}(e)=n_{a b}^{c}(e)=0$.

\section{The action of $G$ on $\mathcal{N}\left(g_{-}, \eta_{-}\right)$}

The group $G$ acts on itself by left translations, $L: G \times G \longrightarrow G$ as

$$
L_{a} g=a g=a_{+} a_{-} g_{+} g_{-},
$$

for $a_{+}, g_{+} \in G_{+}$, and $a_{-}, g_{-} \in G_{-}$. It can be easily lifted to the cotangent bundle $T^{*} G \cong G \times \mathfrak{g}^{*}$, in body coordinates, as the Hamiltonian action

$$
\rho: G \times\left(G \times \mathfrak{g}^{*}\right) \longrightarrow G \times \mathfrak{g}^{*} / \rho_{h}(g, \eta)=(h g, \eta)
$$

with associated $A d$-equivariant momentum map $\Phi^{L}: G \times \mathfrak{g}^{*} \longrightarrow \mathfrak{g}^{*}$,

$$
\Phi^{L}(g, \eta)=A d_{g^{-1}}^{G *} \eta \text {. }
$$


So, the map

$$
\phi_{X}(g, \eta)=\left\langle\eta, A d_{g^{-1}}^{G} X\right\rangle
$$

is the Hamiltonian function associated with the infinitesimal generator $X_{G \times \mathfrak{g}^{*}}$ corresponding to $X \in \mathfrak{g}$, namely, $\iota_{X_{G \times \mathfrak{g}^{*}}} \omega_{\circ}=d \phi_{X}$.

We now use the Dirac bracket (20) to get the Hamiltonian vector field $X_{\mathcal{N}}$ on $\mathcal{N}\left(g_{-}, \eta_{-}\right)$, associated with $X \in \mathfrak{g}$, such that

$$
\left\langle d \mathcal{F}, X_{\mathcal{N}}\right\rangle_{(g, \eta)}:=\left\{\mathcal{F}, \phi_{X}\right\}^{D}(g, \eta) .
$$

Writing $d \phi_{X}=\left(\mathbf{d} \phi_{X}, \delta \phi_{X}\right) \in T^{*} G \oplus T^{*} \mathfrak{g}^{*}$

$$
\left(g \mathbf{d} \phi_{X}, \delta \phi_{X}\right)=\left(\left[\eta, A d_{g^{-1}}^{G} X\right], A d_{g^{-1}}^{G} X\right),
$$

the Poisson-Dirac bracket turns into

$$
\begin{aligned}
\left\{\mathcal{F}, \phi_{X}\right\}^{D}(g, \eta)= & \left\langle g \mathbf{d} \mathcal{F}, A d_{g_{-}^{-1}}^{G} \Pi_{\mathfrak{g}_{+}^{\circ}} A d_{g_{+}^{-1}}^{G} X\right\rangle \\
& -\left\langle\delta \mathcal{F},\left[\eta, A d_{g_{-}^{-1}}^{G} \Pi_{\mathfrak{g}_{-}^{\circ}} A d_{g_{+}^{-1}}^{G} X\right]\right\rangle \\
& +\left\langle\delta \mathcal{F}, A d_{g_{-}^{-1}}^{G} \Pi_{\mathfrak{g}_{+}^{\circ}} A d_{g_{-}}^{G}\left[\eta, A d_{g_{-}^{-1}}^{G} \Pi_{\mathfrak{g}_{-}^{\circ}} A d_{g_{+}^{-1}}^{G} X\right]\right\rangle,
\end{aligned}
$$

and from it we get the Hamiltonian vector field associated with $\phi_{X}$,

$$
\begin{aligned}
X_{\mathcal{N}}(g, \eta) & =V_{\phi_{X}}^{\mathcal{N}\left(g_{-}, \eta_{-}\right)}(g, \eta) \\
& =\left(g A d_{g_{-}^{-1}}^{G} \Pi_{\mathfrak{g}_{+}^{\circ}} A d_{g_{+}^{-1}}^{G} X, A d_{g_{-}^{-1}}^{G} \Pi_{\mathfrak{g}_{-}^{\circ}}\left[\Pi_{\mathfrak{g}_{-}^{\circ}} A d_{g_{+}^{-1}}^{G} X, A d_{g_{-}}^{G} \eta\right]\right) .
\end{aligned}
$$

These vector fields are the projection on $T \mathcal{N}\left(g_{-}, \eta_{-}\right)$of the infinitesimal generators $X_{G \times \mathfrak{g}^{*}}$ of the action of $G$ on $G \times \mathfrak{g}^{*}$ associated with $X \in \mathfrak{g}$. However, it is not clear at this point whether these vector fields are infinitesimal generators for an action of $G$ on $\mathcal{N}\left(g_{-}, \eta_{-}\right)$. This question is addressed in the following proposition.

Proposition: The assignment $X \in \mathfrak{g} \longrightarrow X_{\mathcal{N}} \in \mathfrak{X}\left(\mathcal{N}\left(g_{-}, \eta_{-}\right)\right)$defines a Hamiltonian action of the Lie algebra $\mathfrak{g}$ on $\mathcal{N}\left(g_{-}, \eta_{-}\right)$provided $\eta_{-}$is a character of $\mathfrak{g}_{-}^{\circ}$.

Proof: The Dirac bracket of the Hamiltonian functions $\phi_{X}, \phi_{Y}$ is

$$
\left\{\phi_{X}, \phi_{Y}\right\}^{D}(g, \eta)=\phi_{[X, Y]}(g, \eta)-\left\langle A d_{g_{-}^{*}}^{*} \eta_{-},\left[\Pi_{\mathfrak{g}_{-}^{\circ}} A d_{g_{+}^{-1}}^{G} X, \Pi_{\mathfrak{g}_{-}^{\circ}} A d_{g_{+}^{-1}}^{G} Y\right]\right\rangle,
$$

where it is obvious that the second term in the rhs vanishes for every $X$ and $Y$ in $\mathfrak{g}$ only if $\eta_{-} i s$ character of $\mathfrak{g}_{-}^{\circ}$. In this case, it is easy to see using the Jacobi identity that, for an arbitrary function $f$ on $G \times \mathfrak{g}^{*}$,

$$
\begin{aligned}
\left(\mathbf{L}_{Y_{\mathcal{N}}} \mathbf{L}_{X_{\mathcal{N}}}-\mathbf{L}_{X_{\mathcal{N}}} \mathbf{L}_{Y_{\mathcal{N}}}\right) f(g, \eta)= & \left\{\left\{f, \phi_{X}\right\}^{D}, \phi_{Y}\right\}^{D}(g, \eta) \\
& +\left\{\left\{\phi_{Y}, f\right\}^{D}, \phi_{X}\right\}^{D}(g, \eta) \\
= & \mathbf{L}_{[X, Y]_{\mathcal{N}}} f(g, \eta),
\end{aligned}
$$

that is equivalent to say that the assignment $X \in \mathfrak{g} \longrightarrow X_{\mathcal{N}} \in \mathfrak{X}\left(\mathcal{N}\left(g_{-}, \eta_{-}\right)\right)$is an antihomomorphism of Lie algebras

$$
\left[X_{\mathcal{N}}, Y_{\mathcal{N}}\right]=-[X, Y]_{\mathcal{N}}
$$

Therefore, it defines a left action of $\mathfrak{g}$ on $\mathcal{N}\left(g_{-}, \eta_{-}\right)$.

In particular, at $\eta_{-}=0$ and $g_{-}=e$, we get

$$
X_{\mathcal{N}}\left(g_{+}, \eta_{+}\right)=\left(g_{+}\left(\Pi_{\mathfrak{g}_{+}^{\circ}} A d_{g_{+}^{-1}}^{G} X\right),-\Pi_{\mathfrak{g}_{-}^{\circ}}\left[\eta, \Pi_{\mathfrak{g}_{-}^{\circ}} A d_{g^{-1}}^{G} X\right]\right) .
$$


Observe that, for $X \in\left\{T^{a}\right\}_{a=1}^{n} \subset \mathfrak{g}_{-}^{\circ}$, the momentum functions $\phi^{a}:=\phi_{T^{a}}$ generate the infinitesimal dressing action of $\mathfrak{g}_{-}^{\circ}$ on $\mathcal{N}\left(g_{-}, \eta_{-}\right)$.

This infinitesimal action of $\mathfrak{g}$ on $\mathcal{N}\left(g_{-}, \eta_{-}\right)$corresponds to the following action of $G$ on $\mathcal{N}\left(g_{-}, \eta_{-}\right)$.

Proposition: The vector field $X_{\mathcal{N}} \in \mathfrak{X}\left(\mathcal{N}\left(g_{-}, \eta_{-}\right)\right)$, for $X \in \mathfrak{g}$ and $\eta_{-}$a character of $\mathfrak{g}_{-}^{\circ}$, is the infinitesimal generator associated with the action $G \times \mathcal{N}\left(g_{-}, \eta_{-}\right) \longrightarrow \mathcal{N}\left(g_{-}, \eta_{-}\right)$defined as

$$
\begin{aligned}
& \mathrm{d}(h,(g, \eta))=\left(g A d_{g_{-}^{-1}}^{G} \Pi_{\mathfrak{g}_{+}^{\circ}}\left(g_{+}^{-1} h g_{+}\right), A d_{g_{-}^{-1}}^{G} \Pi_{\mathfrak{g}_{-}^{\circ}} A d_{\Pi_{G_{-}}\left(g_{+}^{-1} h g_{+}\right.}^{G} A d_{g_{-}}^{G} \eta\right), \\
& \forall(g, \eta)=\left(g_{+} g_{-}, \eta_{+}+\eta_{-}\right) \in \mathcal{N}\left(g_{-}, \eta_{-}\right) .
\end{aligned}
$$

Proof: It follows by straightforward calculation of the differential of this map.

Observe that it can be written as

$$
\begin{aligned}
& \mathrm{d}\left(h,\left(g_{+} g_{-}, \eta_{+}+\eta_{-}\right)\right) \\
& =\left(h_{+} g_{+}^{h_{-}} g_{-}, A d_{g_{-}^{-1}}^{G}\left[\left(h_{-}^{g_{+}}\right)^{\eta_{-}^{h_{-}}}\left(h_{-}^{g_{+}}\right)^{-1}+A d_{h_{-}^{g+}}^{G} g_{-}^{\eta_{-}} g_{-}^{-1}\right]\right. \\
& \left.+A d_{g_{-}^{-1} h_{-}^{g_{+}} g_{-}} \eta_{+}+\eta_{-}\right),
\end{aligned}
$$

so that for $g_{-}=e$ and $\eta_{-}=0$, it turns into

$$
\mathrm{d}\left(h,\left(g_{+}, \eta_{+}\right)\right)=\left(h_{+} g_{+}^{h_{-}}, A d_{h_{-}^{g+}} \eta_{+}\right) .
$$

This action was introduced in Ref. 4 as the fundamental ingredient underlying the Poisson Lie T-duality scheme.

Note: The submanifolds we refer above are particular members of a bigger family of symplectic submanifolds in $G \times \mathfrak{g}^{*}$, which are $G$-spaces and can be constructed by means of reduction theory. ${ }^{10}$ In this framework, the procedure shows, in particular, that $\mathcal{N}\left(g_{-}, \eta_{-}\right)$and $\mathcal{M}\left(g_{+}, \eta_{+}\right)$are symplectic submanifolds of $G \times \mathfrak{g}^{*}$ if $\eta_{ \pm}$are characters of the coadjoint action of $G$ on $\mathfrak{g}^{*}$, respectively. Additionally, it gives an interpretation for the actions of the factorizable Lie group $G=G_{+} G_{-}$on $\mathcal{N}\left(g_{-}, \eta_{-}\right)$and $\mathcal{M}\left(g_{+}, \eta_{+}\right)$, providing us an explanation to the symplecticity of these actions. For instance, in the case of $\mathcal{N}\left(g_{-}, \eta_{-}\right)$we can think of it as follows: the right action of $G_{-}$on $G$ induces on $G \times \mathfrak{g}^{*}$ a symplectic action by lifting and by applying the Marsden-Weinstein reduction via its momentum map $J_{-}: G \times \mathfrak{g}^{*} \rightarrow \mathfrak{g}_{-}^{*} \simeq \mathfrak{g}_{+}^{\circ}$, for $\eta_{-} \in \mathfrak{g}_{+}^{\circ}$, we get the quotient map

$$
\pi_{\eta_{-}}: J_{-}^{-1}\left(\eta_{-}\right) \rightarrow J_{-}^{-1}\left(\eta_{-}\right) /\left(G_{-}\right)_{\eta_{-}}
$$

such that the symplectic form $\omega_{\eta_{-}}$on $J_{-}^{-1}\left(\eta_{-}\right) /\left(G_{-}\right)_{\eta_{-}}$is defined by the condition

$$
\pi_{\eta_{-}}^{*} \omega_{\eta_{-}}=\omega_{\circ} \mid\left(J_{-}\right)^{-1}\left(\eta_{-}\right)
$$

where $\omega_{\circ}$ indicates the canonical 2-form on $G \times \mathfrak{g}^{*}$. In this context, we have the following remarkable facts:

- for $\eta_{-} \in \mathfrak{g}_{+}^{\circ}$ a character, we have that $\left(G_{-}\right)_{\eta_{-}}=G_{-}$for its isotropy group,

- any submanifold $S \subset J_{-}^{-1}\left(\eta_{-}\right)$transverse to the $G_{-}$-orbits and such that $\operatorname{dim} S$ $=\operatorname{dim}\left[J_{-}^{-1}\left(\eta_{-}\right) / G_{-}\right]$(i.e., $S$ is a cross section for the $G_{-}$-action on $J_{-}^{-1}\left(\eta_{-}\right)$), is a symplectic submanifold of $G \times \mathfrak{g}^{*}$ via the restriction of the canonical 2-form, and

- $\pi_{\eta_{-}} \mid S$ is a symplectomorphism.

By viewing $\mathcal{N}\left(g_{-}, \eta_{-}\right)$( $\eta_{-}$a character!) as submanifolds of $J_{-}^{-1}\left(\eta_{-}\right)$transverse to the $G_{-}$-orbits, we find that they are symplectic submanifolds of $G \times \mathfrak{g}^{*}$ and symplectomorphic to the Marsden-Weinstein reduced space $J_{-}^{-1}\left(\eta_{-}\right) / G_{-}$. 
On the other side, it can be proved that the Marsden-Weinstein reduced space obtained above is symplectomorphic to the cotangent bundle of a (reductive) homogeneous space $M$ of $G$, and this fact has the following two consequences:

- any slice in $J_{-}^{-1}\left(\eta_{-}\right)$is symplectomorphic to $T^{*} M$, and

- the lifted canonical action of $G$ on $T^{*} M$ induces a symplectic action on these slices.

We see that the action of $G$ on $\mathcal{N}\left(g_{-}, \eta_{-}\right)$defined above (24) comes from this construction. Summarizing, we can fit all the relevant structures in the following diagram:

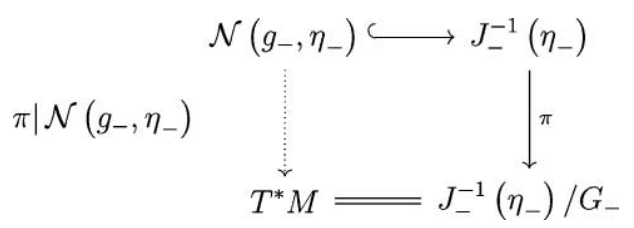

The dotted arrow can be viewed as a consequence of the rest of the structures in the diagram, inducing the action while keeps it symplectic. The particular case $\left(g_{-}, \eta_{-}\right)=(e, 0)$ gives the $G$-action on $G_{+}$ considered in Ref. 4.

\section{The Hamilton equations on $\mathcal{N}\left(g_{-}, \eta_{-}\right)$}

Let us consider a generic Hamiltonian function $\mathcal{H}$ on $G \times \mathfrak{g}^{*}$, so the associated Hamiltonian vector field by the Poisson-Dirac structure (20) is

$$
\begin{aligned}
& V_{\mathcal{H}}^{\mathcal{N}}(g, \eta) \\
& =\left(g A d_{g_{-}^{-1}}^{G} \Pi_{\mathfrak{g}_{+}^{\circ}} A d_{g_{-}}^{G} \delta \mathcal{H}, A d_{g_{-}^{-1}}^{G} \Pi_{\mathfrak{g}_{-}^{\circ}} A d_{g_{-}}^{G}\left(\left[\eta, A d_{g_{-}^{-1}}^{G} \Pi_{\mathfrak{g}_{+}^{\circ}} A d_{g_{-}}^{G} \delta \mathcal{H}\right]-g \mathbf{d} \mathcal{H}\right)\right)
\end{aligned}
$$

and the Hamilton equation are

$$
\left\{\begin{array}{l}
g^{-1} \dot{g}=\left(A d_{g_{-}^{-1}}^{G} \Pi_{\mathfrak{g}_{+}^{\circ}} A d_{g_{-}}^{G}\right) \delta \mathcal{H} \\
\dot{\eta}=\left(A d_{g_{-}^{-1}}^{G} \Pi_{\mathfrak{g}_{-}^{\circ}} A d_{g_{-}}^{G}\right)\left(\left[\eta, A d_{g_{-}^{-1}}^{G} \Pi_{\mathfrak{g}_{+}^{\circ}} A d_{g_{-}}^{G} \delta \mathcal{H}\right]-g \mathbf{d} \mathcal{H}\right) .
\end{array}\right.
$$

In terms of the factors, $g=g_{+} g_{-}$and $\eta=\eta_{+}+\eta_{-}$, they are equivalent to

$$
\begin{aligned}
& \left\{\begin{array}{l}
g_{+}^{-1} \dot{g}_{+}=\Pi_{\mathfrak{g}_{+}^{\circ}} A d_{g_{-}}^{G} \delta \mathcal{H} \\
\dot{\eta}_{+}=A d_{g_{-}^{-1}}^{G} \Pi_{\mathfrak{g}_{-}^{\circ}} A d_{g_{-}}^{G}\left(\left[\eta, A d_{g_{-}^{-1}}^{G} \Pi_{\mathfrak{g}_{+}^{\circ}} A d_{g_{-}}^{G} \delta \mathcal{H}\right]-g \mathbf{d} \mathcal{H}\right),
\end{array}\right. \\
& \left\{\begin{array}{l}
\dot{g}_{-} g_{-}^{-1}=0 \\
\dot{\eta}_{-}=0
\end{array}\right.
\end{aligned}
$$

By using the fundamental coordinates, the Hamilton equation on $\mathcal{N}\left(g_{-}, \eta_{-}\right)$are

$$
\left\{\begin{array}{l}
\dot{\mathrm{T}}_{j}^{k}=\left\{\mathrm{T}_{j}^{k}, \mathcal{H}\right\}^{D} \\
\dot{\xi}_{a}=\left\{\xi_{a}, \mathcal{H}\right\}^{D}
\end{array}\right.
$$

that turn out to be

$$
\left\{\begin{array}{l}
\dot{\mathrm{T}}_{j}^{k}=\frac{\partial \mathcal{H}}{\partial \xi_{b}}\left\{\mathrm{~T}_{j}^{k}, \xi_{b}\right\}^{D} \\
\dot{\xi}_{a}=\frac{\partial \mathcal{H}}{\partial \mathrm{T}_{i}^{j}}\left\{\xi_{a}, \mathrm{~T}_{i}^{j}\right\}^{D}+\frac{\partial \mathcal{H}}{\partial \xi_{b}}\left\{\xi_{a}, \xi_{b}\right\}^{D}
\end{array}\right.
$$




\section{B. The phase spaces $\mathcal{M}\left(g_{+}, \eta_{+}\right)$}

Let us consider the fibration $\Upsilon: G \times \mathfrak{g}^{*} \longrightarrow G_{+} \times \mathfrak{g}_{+}^{*}$ with fiber $\mathcal{M}\left(g_{+}, \eta_{-}\right)$at $\left(g_{+}, \eta_{-}\right)$ $\in G_{+} \times \mathfrak{g}_{+}^{*}$. The differential of the projection of a vector $(v, \xi) \in G \times \mathfrak{g}^{*}$ on $G_{+} \times \mathfrak{g}_{+}^{*}$ is

$$
\Pi_{+*}(v, \xi)=\left(g_{+} A d_{g_{-}^{-1}}^{*} X_{+}, \xi_{+}\right)_{\left(g_{+}, \eta_{+}\right)},
$$

where $X_{+}=\Pi_{\mathfrak{g}_{+}^{\circ}} g^{-1} v$ and $\xi=\xi_{+}+\xi_{-}$. Hence, coming back to the expression for $\Upsilon_{*}$, we have

$$
\left.\Upsilon_{*}(g X, \xi)\right|_{(g, \eta)}=\left(g_{+} A d_{g_{-}^{-1}}^{*} X_{+}, \xi_{+}\right)_{\left(g_{+}, \eta_{+}\right)} .
$$

Let us find out $\left.\operatorname{ker} \Upsilon_{*}\right|_{(g, \eta)}=T_{(g, \eta)} \mathcal{M}\left(g_{+}, \eta_{+}\right)$, using (13) we get

$$
\left.\operatorname{ker} \Upsilon_{*}\right|_{(g, \eta)}=\left\{\left(g X_{-}, \xi_{-}\right) /\left(X_{-}, \xi_{-}\right) \in \mathfrak{g}_{-}^{\circ} \oplus \mathfrak{g}_{-}^{*}\right\} .
$$

We may use this result to analyze the intersection of $\operatorname{ker} \Upsilon_{*}$ with the symplectic orthogonal of $T \Upsilon^{-1}\left(g_{+}, \eta_{+}\right)$. In order to do this, we evaluate

$$
\left\langle\omega_{\circ},\left(g X_{-}, \xi_{-}\right) \otimes\left(g Y_{-}, \lambda_{-}\right)\right\rangle_{(g, \eta)}=-\left\langle\xi_{-}, Y_{-}\right\rangle+\left\langle\lambda_{-}, X_{-}\right\rangle+\left\langle\eta_{-},\left[X_{-}, Y_{-}\right]\right\rangle,
$$

that is regular on $T \mathcal{M}\left(g_{+}, \eta_{+}\right)$, so that $\left.\left[T_{(g, \eta)} \mathcal{M}\left(g_{+}, \eta_{+}\right)\right]^{\omega_{\circ} \perp} \cap \operatorname{ker} \Upsilon_{*}\right|_{(g, \eta)}=\{0\}$.

Proposition: $\left(\mathcal{M}\left(g_{+}, \eta_{+}\right), \hat{\omega}_{\circ}\right)$, where $\hat{\omega}_{\circ}$ stands for the restriction of the canonical symplectic form $\omega_{\circ}$ on $G \times \mathfrak{g}^{*}$ to $\Upsilon^{-1}\left(g_{+}, \eta_{+}\right)$, is a symplectic manifold. Moreover $\hat{\omega}_{\circ}$ coincides with the canonical symplectic form of $G_{-} \times \mathfrak{g}_{-}^{*}$.

Consequently, the restriction to $\Upsilon^{-1}\left(g_{+}, \eta_{+}\right)$is a second class constraint, as expected.

In order to built up the Dirac brackets, we take a set of linearly independent 1-forms on $G_{+} \times \mathfrak{g}_{+}^{*}$,

$$
\begin{aligned}
& \theta_{a}=\left(L_{g_{+}^{-1}}^{*} T^{a}, 0\right) \in T_{\left(g_{+}, \eta_{+}\right)}^{*}\left(G_{+} \times \mathfrak{g}_{+}^{*}\right), \\
& \gamma_{a}=\left(0, T_{a}\right) \in T_{\left(g_{+}, \eta_{+}\right)}^{*}\left(G_{+} \times \mathfrak{g}_{+}^{*}\right) .
\end{aligned}
$$

Then their pullback $\left\{\Upsilon^{*} \theta^{a}, \Upsilon^{*} \gamma_{a}\right\}_{a=1}^{n}$ are a set of linearly independent 1-forms on $G \times \mathfrak{g}^{*}$, such that $\left(\left\{\Upsilon^{*} \theta^{a}, \Upsilon^{*} \gamma_{a}\right\}_{a=1}^{n}\right)^{\circ}=T \mathcal{M}\left(g_{+}, \eta_{+}\right)$. The Hamiltonian vector fields of these 1-forms on $G \times \mathfrak{g}^{*}$ are

$$
\begin{aligned}
& \left.V_{\Upsilon^{*} \theta^{a}}\right|_{(g, \eta)}=\left(0,-A d_{g_{-}^{-1}} T^{a}\right)_{(g, \eta)}, \\
& \left.V_{\Upsilon^{*} \gamma_{a}}\right|_{(g, \eta)}=\left(g T_{a}, a d_{T_{a}}^{\mathfrak{g} *} \eta\right),
\end{aligned}
$$

and the Dirac matrix

$$
C=\left(\begin{array}{cc}
\left\langle\Upsilon^{*} \theta^{a}, V_{\Upsilon^{*} \theta^{b}}\right\rangle_{(g, \eta)} & \left\langle\Upsilon^{*} \theta^{a}, V_{\Upsilon^{*} \gamma_{b}}\right\rangle_{(g, \eta)} \\
\left\langle\Upsilon^{*} \gamma_{a}, V_{\Upsilon^{*} \theta^{b}}\right\rangle_{(g, \eta)} & \left\langle\Upsilon^{*} \gamma_{a}, V_{\Upsilon^{*} \gamma_{b}}\right\rangle_{(g, \eta)}
\end{array}\right)
$$

is

$$
C(g, \eta)=\left[\begin{array}{cc}
0_{n \times n} & F\left(g_{-}\right) \\
-F^{\top}\left(g_{-}\right) & \Theta\left(\eta_{+}\right)
\end{array}\right],
$$

where $\Theta\left(\eta_{+}\right)$is an $n \times n$ matrix with entries

$$
\Theta_{a b}\left(\eta_{+}\right)=\left\{\Upsilon^{*} \gamma_{a}, \Upsilon^{*} \gamma_{b}\right\}\left(\eta_{+}\right)=-\left\langle\eta_{+},\left[T_{a}, T_{b}\right]\right\rangle
$$

and

$$
F_{b}^{a}\left(g_{-}\right)=\left\{\Upsilon^{*} \theta^{a}, \Upsilon^{*} \gamma_{b}\right\}\left(g_{-}\right)=\left\langle A d_{g_{-}^{-1}} T^{a}, T_{b}\right\rangle
$$


The inverse of the Dirac matrix is

$$
C^{-1}(g, \eta)=\left(\begin{array}{cc}
F_{a}^{c}\left(g_{-}^{-1}\right) \Theta_{c d}\left(\eta_{+}\right) F_{b}^{d}\left(g_{-}^{-1}\right) & -F_{a}^{b}\left(g_{-}^{-1}\right) \\
F_{b}^{a}\left(g_{-}^{-1}\right) & 0_{n \times n}
\end{array}\right)
$$

that reduces to

$$
C^{-1}(g, \eta)=\left(\begin{array}{cc}
-\left\langle\eta_{+},\left[A d_{g_{-}}^{*} T_{a}, A d_{g_{-}}^{*} T_{b}\right]\right\rangle & -\left\langle A d_{g_{-}} T^{b}, T_{a}\right\rangle \\
\left\langle A d_{g_{-}} T^{a}, T_{b}\right\rangle & 0_{n \times n}
\end{array}\right) .
$$

We are now ready to construct the Dirac bracket on $\mathcal{M}\left(g_{+}, \eta_{+}\right)$. Introducing this result into (8), we get

$$
\begin{aligned}
\{\mathcal{F}, \mathcal{H}\}^{\mathcal{M}}(g, \eta)= & \left\langle g \mathbf{d} \mathcal{F}, \Pi_{\mathfrak{g}_{-}^{\circ}} \delta \mathcal{H}\right\rangle-\left\langle g \mathbf{d} \mathcal{H}, \Pi_{\mathfrak{g}_{-}^{\circ}} \delta \mathcal{F}\right\rangle \\
& -\left\langle\eta_{-},\left[\Pi_{\mathfrak{g}_{-}^{\circ}} \delta \mathcal{F}, \Pi_{\mathfrak{g}_{-}^{\circ}} \delta \mathcal{H}\right]\right\rangle
\end{aligned}
$$

that coincides with the canonical Poisson bracket on $G_{-} \times \mathfrak{g}_{-}^{*}$.

\section{The left action of $G$ on $G \times \mathfrak{g}^{*}$ and its restriction to $\mathcal{M}\left(g_{+}, \eta_{+}\right)$}

As described at the beginning of Sec. III A 3, we want to study how the left action of $G$ on its cotangent bundle project on the fibers $\mathcal{M}\left(g_{+}, \eta_{+}\right)$. In doing so, we consider the Dirac bracket (26) involving the momentum function $\phi_{X}$, which for an arbitrary function $\mathcal{F} \in C^{\infty}\left(G \times \mathfrak{g}^{*}\right)$ gives

$$
\begin{aligned}
\left\{\mathcal{F}, \phi_{X}\right\}^{\mathcal{M}}(g, \eta)= & \left\langle g \mathbf{d} \mathcal{F}, \Pi_{\mathfrak{g}_{-}^{\circ}} A d_{g^{-1}}^{G} X\right\rangle-\left\langle\left[\eta, A d_{g^{-1}}^{G} X\right], \Pi_{\mathfrak{g}_{-}^{\circ}} \delta \mathcal{F}\right\rangle \\
& -\left\langle\eta_{-},\left[\Pi_{\mathfrak{g}_{-}^{\circ}} \delta \mathcal{F}, \Pi_{\mathfrak{g}_{-}^{\circ}} A d_{g^{-1}}^{G} X\right]\right\rangle,
\end{aligned}
$$

therefore the Hamiltonian vector field of $\phi_{X}$ is

$$
V_{\phi_{X}}^{\mathcal{M}\left(g_{+}, \eta_{+}\right)}(g, \eta)=\left(g \Pi_{\mathfrak{g}_{-}^{\circ}} A d_{g^{-1}}^{G} X, \Pi_{\mathfrak{g}_{+}^{\circ}}\left[\Pi_{\mathfrak{g}_{+}^{\circ}} A d_{g^{-1}}^{G} X, \eta\right]\right) .
$$

On the other side, the Dirac bracket between two moment functions reduces to

$$
\left\{\phi_{X}, \phi_{Y}\right\}^{\mathcal{M}}(g, \eta)=\phi_{[X, Y]}(g, \eta)-\left\langle\eta_{+},\left[\Pi_{\mathfrak{g}_{+}^{\circ}} A d_{g^{-1}}^{G} X, \Pi_{\mathfrak{g}_{+}^{\circ}} A d_{g^{-1}}^{G} Y\right]\right\rangle
$$

so, as it happens on $\mathcal{N}\left(g_{-}, \eta_{-}\right)$, it closes an algebra provided $\eta_{+}$is a character of $\mathfrak{g}_{+}^{\circ}$.

From the expression of the Hamiltonian vector field $V_{\phi_{X}}^{\mathcal{M}\left(g_{+}, \eta_{+}\right)}$given in Eq. (27), we retrieve the action of $G$ on $\mathcal{M}\left(g_{+}, \eta_{+}\right)$, b : $G \times \mathcal{M}\left(g_{+}, \eta_{+}\right) \longrightarrow \mathcal{M}\left(g_{+}, \eta_{+}\right)$, in the case $\eta_{+}$is a character of $\mathfrak{g}_{+}^{\circ}$,

$$
\mathrm{b}\left(h,\left(g_{+} g_{-}, \eta_{+}+\eta_{-}\right)\right)=\left(g \Pi_{-}\left(g^{-1} h g\right), \Pi_{\mathfrak{g}_{+}^{\circ}} A d_{\Pi_{-}\left(g^{-1} h g\right)}^{G} \eta\right) .
$$

\section{The Hamilton equations}

The Hamilton equations on $\mathcal{M}\left(g_{+}, \eta_{+}\right)$are defined by the Dirac bracket (26),

$$
\{\mathcal{F}, \mathcal{H}\}^{\mathcal{M}}(g, \eta)=\left\langle g \mathbf{d} \mathcal{F}, \Pi_{\mathfrak{g}_{-}^{\circ}} \delta \mathcal{H}\right\rangle-\left\langle g \mathbf{d} \mathcal{H}, \Pi_{\mathfrak{g}_{-}^{\circ}} \delta \mathcal{F}\right\rangle-\left\langle\eta_{-},\left[\Pi_{\mathfrak{g}_{-}^{\circ}} \delta \mathcal{F}, \Pi_{\mathfrak{g}_{-}^{\circ}} \delta \mathcal{H}\right]\right\rangle,
$$

from where we get the Hamiltonian vector field for the Hamilton function $\mathcal{H}$,

$$
V_{\mathcal{H}}^{\mathcal{M}}(g, \eta)=\left(g \Pi_{\mathfrak{g}_{-}^{\circ}} \delta \mathcal{H}, \Pi_{\mathfrak{g}_{+}^{\circ}}\left(g \mathbf{d} \mathcal{H}-\left(\left[\eta_{-}, \Pi_{\mathfrak{g}_{-}^{\circ}} \delta \mathcal{H}\right]\right)\right)\right) .
$$


The reduced Hamilton equations are then

$$
\left\{\begin{array}{l}
g^{-1} \dot{g}=\Pi_{\mathfrak{g}_{-}^{\circ}} \delta \mathcal{H} \\
\dot{\eta}=\Pi_{\mathfrak{g}_{+}^{\circ}}\left(g \mathbf{d} \mathcal{H}-\left(\left[\eta_{-}, \Pi_{\mathfrak{g}_{-}^{\circ}} \delta \mathcal{H}\right]\right)\right)
\end{array}\right.
$$

that, when expressed in terms of the factors, $g=g_{+} g_{-}$and $\eta=\eta_{+}+\eta_{-}$, becomes in

$$
\begin{aligned}
& \left\{\begin{array}{l}
g_{-}^{-1} \dot{g}_{-}=\Pi_{\mathfrak{g}_{-}^{\circ}} \delta \mathcal{H} \\
\dot{\eta}_{-}=\Pi_{\mathfrak{g}_{+}^{\circ}}\left(g \mathbf{d} \mathcal{H}-\left(\left[\eta_{-}, \Pi_{\mathfrak{g}_{-}^{\circ}} \delta \mathcal{H}\right]\right)\right),
\end{array}\right. \\
& \left\{\begin{array}{l}
g_{+}^{-1} \dot{g}_{+}=0 \\
\dot{\eta}_{+}=0 .
\end{array}\right.
\end{aligned}
$$

\section{THE DIRAC METHOD AND INTEGRABLE SYSTEMS}

\section{A. Involutive function algebra in $\mathcal{N}\left(e, \eta_{-}\right)$}

The vector space $\mathfrak{g}^{*}$ turns into a Poisson manifold provided we equip the set $C^{\infty}\left(\mathfrak{g}^{*}\right)$ with one of the Kirillov-Kostant bracket $\{,\}_{ \pm}: C^{\infty}\left(\mathfrak{g}^{*}\right) \times C^{\infty}\left(\mathfrak{g}^{*}\right) \longrightarrow C^{\infty}\left(\mathfrak{g}^{*}\right)$ defined as

$$
\{\mathrm{f}, \mathrm{g}\}_{-}(\eta)=-\left\langle\eta,\left[\mathcal{L}_{\mathrm{f}}(\eta), \mathcal{L}_{\mathrm{g}}(\eta)\right]\right\rangle,
$$

where $\mathcal{L}_{\mathrm{h}}: \mathfrak{g}^{*} \longrightarrow \mathfrak{g}$ stands for the Legendre transformation of a function $\mathrm{h}: \mathfrak{g}^{*} \longrightarrow \mathbb{R}$, such that for any $\xi \in \mathfrak{g}^{*}$,

$$
\left\langle\xi, \mathcal{L}_{\mathrm{h}}(\eta)\right\rangle_{\mathfrak{g}}=\left\langle\left. d \mathrm{~h}\right|_{\eta}, \xi\right\rangle_{\mathfrak{g}^{*}}=\left.\frac{d \mathrm{~h}(\eta+t \xi)}{d t}\right|_{t=0} .
$$

The canonical Poisson bracket on $G \times \mathfrak{g}^{*}$,

$$
\{\mathcal{F}, \mathcal{G}\}(g, \eta)=\langle\mathbf{d} \mathcal{F}, g \delta \mathcal{G}\rangle-\langle\mathbf{d} \mathcal{G}, g \delta \mathcal{F}\rangle-\langle\eta,[\delta \mathcal{F}, \delta \mathcal{G}]\rangle
$$

for $\mathcal{F}, \mathcal{G}=\mathfrak{f}, \mathbf{g} \in C^{\infty}\left(\mathfrak{g}^{*}\right)$ such that $\left.d \mathfrak{f}\right|_{\eta}=\left.(0, \delta \mathfrak{f})\right|_{\eta}=\left(0, \mathcal{L}_{\mathfrak{f}}(\eta)\right)$, it reduces to

$$
\{\mathrm{f}, \mathrm{g}\}(g, \eta)=-\left\langle\eta,\left[\mathcal{L}_{\mathrm{f}}(\eta), \mathcal{L}_{\mathrm{g}}(\eta)\right]\right\rangle .
$$

A remarkable fact here is that the symplectic leaves of this Poisson structure coincides with the orbits of the coadjoint action of $G$ on $\mathfrak{g}^{*}$.

Let us now study the constraint submanifold $\mathcal{N}\left(e, \eta_{-}\right)$, equipped with Poisson-Dirac structure derived from the Dirac bracket (20),

$$
\{\mathcal{F}, \mathcal{G}\}^{\mathcal{N}}\left(g_{+}, \eta\right)=\left\langle g \mathbf{d} \mathcal{F}, \Pi_{\mathfrak{g}_{+}^{\circ}} \delta \mathcal{G}\right\rangle-\left\langle g \mathbf{d} \mathcal{G}, \Pi_{\mathfrak{g}_{+}^{\circ}} \delta \mathcal{F}\right\rangle-\left\langle\eta,\left[\Pi_{\mathfrak{g}_{+}^{\circ}} \delta \mathcal{F}, \Pi_{\mathfrak{g}_{+}^{\circ}} \delta \mathcal{G}\right]\right\rangle
$$

which, when applied to $f, g C^{\infty}\left(\mathfrak{g}^{*}\right)$, gives

$$
\{\mathbf{f}, \mathbf{g}\}^{\mathcal{N}}\left(g_{+}, \eta\right)=-\left\langle\eta,\left[\Pi_{\mathfrak{g}_{+}^{\circ}} \mathcal{L}_{\mathfrak{f}}(\eta), \Pi_{\mathfrak{g}_{+}^{\circ}} \mathcal{L}_{\mathbf{g}}(\eta)\right]\right\rangle,
$$

meaning that $\mathcal{N}\left(e, \eta_{-}\right)$is equipped with a kind of Kirillov-Kostant bracket just as $\mathfrak{g}_{+}^{*}$, where $\eta_{-}$ enters as a parameter in the Legendre transform of the functions $f, g \in C^{\infty}\left(\mathfrak{g}^{*}\right)$. Then, for $f, g$ being $A d^{G}$-invariant functions we have the relation

$$
\mathcal{L}_{\mathrm{h}}\left(A d_{g}^{*} \eta\right)=A d_{g-1} \mathcal{L}_{\mathrm{h}}(\eta)
$$

that infinitesimally is $\left[\mathcal{L}_{\mathrm{f}}(\eta), \eta\right]=0$, therefore we may also write

$$
\{\mathbf{f}, \mathbf{g}\}^{\mathcal{N}}\left(g_{+}, \eta\right)=\left\langle\eta,\left[\Pi_{\mathfrak{g}_{-}^{\circ}} \mathcal{L}_{\mathfrak{g}}(\eta), \Pi_{\mathfrak{g}_{-}^{\circ}} \mathcal{L}_{\mathfrak{f}}(\eta)\right]\right\rangle .
$$

Hence, provided $\eta_{-}$is a character of $\mathfrak{g}_{-}^{\circ}$, we get

$$
\{\mathrm{f}, \mathrm{g}\}^{\mathcal{N}}\left(g_{+}, \eta\right)=0,
$$


meaning that $\mathrm{f}, \mathrm{g}$ are involutive in relation to the Dirac bracket which restricts them to the submanifold $\mathcal{N}\left(e, \eta_{-}\right)$. For the special value $\eta_{-}=0$, it is just the $A K S$ result. ${ }^{1}$

\section{B. Solving a system in $\mathcal{N}\left(g_{-}, \eta_{-}\right)$by factorization}

Let us now consider the collective Hamiltonian $\mathrm{h} \circ \Phi^{L}: G \times \mathfrak{g}^{*} \longrightarrow \mathbb{R}$, with $\mathrm{h}$ being $A d^{G}$-invariant as above, and $\Phi^{L}$ the momentum map associated with the left translation symmetry given in Eq. (22). In this way, the Hamiltonian function is bi-invariant. Since

$$
d\left(\mathrm{~h} \circ \Phi^{L}\right)=d \mathrm{~h} \circ \Phi_{*}^{L}=\delta \mathrm{h} \circ \Phi_{*}^{L},
$$

if $\mathcal{L}_{\mathrm{h}}: \mathfrak{g}^{*} \longrightarrow \mathfrak{g}$ is the Legendre transformation of $\mathrm{h}$, the differential of the Hamilton function $\mathrm{h} \circ \Phi^{L}$ reduces to

$$
\left.d\left(\mathrm{~h} \circ \Phi^{L}\right)\right|_{(g, \eta)}=\left(0, \mathcal{L}_{\mathrm{h}}\left(A d_{g}^{*} \Phi^{L}(g, \eta)\right)\right)=\left(0, \mathcal{L}_{\mathrm{h}}(\eta)\right),
$$

and the associated Hamiltonian vector field on $G \times \mathfrak{g}^{*}$ is

$$
V_{\mathrm{h} \circ \Phi^{L}}=\left(g \mathcal{L}_{\mathrm{h}}(\eta), 0\right) \text {. }
$$

With this result, we evaluate the Hamiltonian vector field using the Dirac bracket $\left\{\mathcal{F}, \mathrm{h} \circ \Phi^{L}\right\}^{N}(g, \eta)$ defined in (20), obtaining

$$
V_{\mathrm{h}^{\circ} \Phi^{L}}^{N}=\left(g A d_{g_{-}^{-1}}^{G} \Pi_{\mathfrak{g}_{+}^{\circ}} A d_{g_{-}}^{G} \mathcal{L}_{\mathrm{h}}(\eta), A d_{g_{-}^{-1}} \Pi_{\mathfrak{g}_{-}^{\circ}} A d_{g_{-}}^{G}\left[\eta, A d_{g_{-}^{-1}}^{G} \Pi_{\mathfrak{g}_{+}^{\circ}} A d_{g_{-}}^{G} \mathcal{L}_{\mathrm{h}}(\eta)\right]\right) .
$$

Therefore, the Hamilton equations motion are

$$
\left\{\begin{array}{l}
g^{-1} \dot{g}=A d_{g_{-}^{-1}}^{G} \Pi_{\mathfrak{g}_{+}^{\circ}} A d_{g_{-}}^{G} \mathcal{L}_{\mathrm{h}}(\eta) \\
\dot{\eta}=A d_{g_{-}^{-1}} \Pi_{\mathfrak{g}_{-}^{\circ}} A d_{g_{-}}^{G}\left[\eta, A d_{g_{-}^{-1}}^{G} \Pi_{\mathfrak{g}_{+}^{\circ}} A d_{g_{-}}^{G} \mathcal{L}_{\mathrm{h}}(\eta)\right],
\end{array}\right.
$$

that in terms of $g=g_{+} g_{-}$and $\eta=\eta_{+}+\eta_{-}$gives rise to the

$$
\begin{aligned}
& \left\{\begin{array}{l}
g_{+}^{-1} \dot{g}_{+}=\Pi_{\mathfrak{g}_{+}^{\circ}} A d_{g_{-}}^{G} \mathcal{L}_{\mathrm{h}}(\eta) \\
\dot{\eta}_{+}=-A d_{g_{-}^{-1}} \Pi_{\mathfrak{g}_{-}^{\circ}}\left[\Pi_{\mathfrak{g}_{+}^{\circ}} A d_{g_{-}}^{G} \mathcal{L}_{\mathrm{h}}(\eta), \Pi_{\mathfrak{g}_{-}^{\circ}} A d_{g_{-}}^{G} \eta\right],
\end{array}\right. \\
& \left\{\begin{array}{l}
\dot{g}_{-} g_{-}^{-1}=0 \\
\dot{\eta}_{-}=0
\end{array}\right.
\end{aligned}
$$

Introducing $\lambda=A d_{g_{-}}^{G} \eta$ so, if $\eta_{-}$is a character of $\mathfrak{g}_{-}^{\circ}$,

$$
\lambda_{-}=\Pi_{\mathfrak{g}_{+}^{\circ}} A d_{g_{-}}^{G} \eta_{+}+\Pi_{\mathfrak{g}_{+}^{\circ}} A d_{g_{-}}^{G} \eta_{-}=A d_{g_{-}^{-1}}^{*} \eta_{-}=\eta_{-},
$$

here, $\lambda_{-}$is also a character of $\mathfrak{g}_{-}^{\circ}$, the first couple of equation turns in

$$
\left\{\begin{array}{l}
g_{+}^{-1} \dot{g}_{+}=\Pi_{\mathfrak{g}_{+}^{\circ}} \mathcal{L}_{\mathrm{h}}(\lambda) \\
\dot{\lambda}_{+}=-\Pi_{\mathfrak{g}_{-}^{\circ}}\left[\Pi_{\mathfrak{g}_{+}^{\circ}} \mathcal{L}_{\mathrm{h}}(\lambda), \lambda\right] .
\end{array}\right.
$$

Because the $A d$-invariance of $\mathrm{h}$ and having in mind that $\lambda_{-}$is a character of $\mathfrak{g}_{-}^{\circ}$, the second equation is equivalent to

$$
\dot{\lambda}_{+}=a d_{\Pi_{\mathfrak{g}_{-}} \mathcal{L}_{\mathrm{h}}(\lambda)}^{\mathfrak{g}} \lambda
$$

and because $\lambda_{-}=\eta_{-}=$const, we may write

$$
\dot{\lambda}=a d_{\Pi_{\mathfrak{g}_{-}^{\circ}} \mathcal{L}_{\mathrm{h}}(\lambda)}^{\mathfrak{g}} \lambda .
$$


Introducing the curve $h_{-}(t) \subset G_{-}$satisfying the differential equation

$$
\dot{h}_{-} h_{-}^{-1}=\Pi_{\mathfrak{g}_{-}^{\circ}} \mathcal{L}_{\mathrm{h}}(\lambda),
$$

we see that

$$
g_{+}^{-1} \dot{g}_{+}+\dot{h}_{-} h_{-}^{-1}=\mathcal{L}_{\mathrm{h}}(\lambda) .
$$

Let us now write $\xi=A d_{h_{-}^{-1}}^{G} \lambda$ so, the last equation is

$$
A d_{g_{-}^{-1}}^{G}\left(g_{+}^{-1} \dot{g}_{+}+\dot{h}_{-} h_{-}^{-1}\right)=\mathcal{L}_{\mathrm{h}}\left(A d_{h_{-}}^{G} \xi\right)
$$

that can be written as

$$
k^{-1}(t) \dot{k}(t)=\mathcal{L}_{\mathrm{h}}(\xi(t))
$$

where $k(t):=g_{+}(t) h_{-}(t)$.

Having in mind Eqs. (34) and (33), we get

$$
\dot{\xi}=0 .
$$

Thus, the couple of equations

$$
\left\{\begin{array}{l}
k^{-1} \dot{k}=\mathcal{L}_{\mathrm{h}}(\xi) \\
\dot{\xi}=0
\end{array}\right.
$$

are solved by the curves

$$
\left\{\begin{array}{l}
k(t)=e^{t \mathcal{L}_{\mathrm{h}}(\xi)} \\
\xi=\xi_{\circ}
\end{array}\right.
$$

Therefore, we have shown that the Hamilton equations (32) on $\mathcal{N}\left(g_{-}, \eta_{-}\right)$have the solutions

$$
\left\{\begin{array}{l}
g_{+}(t)=\Pi_{G_{+}} e^{t \mathcal{L}_{\mathrm{h}}(\xi)} \\
\eta(t)=A d_{g_{-}^{-1} \Pi_{G_{-}} e^{t \mathcal{L}_{\mathrm{h}}(\xi)}} \xi_{\circ},
\end{array}\right.
$$

encountering the Adler-Kostant-Symes result, ${ }^{1,11}$ relating a system of differential equations on a coadjoint orbit with the factorization problem of an exponential curve in $G$. This issue deserves a deeper insight which is addressed in the next subsection.

\section{AKS theory}

In order to understand the above results, let us digress on the meaning of the Adler-KostantSymes approach to integrability. ${ }^{111}$ The AKS systems can be characterized as reduced spaces derived from a dynamical systems defined on the cotangent bundle of a Lie group, so our main concern in this section will be to provide a connection between this kind of systems and the dynamical systems studied above on the phase spaces $\mathcal{N}\left(g_{-}, \eta_{-}\right)$(analogous considerations can be made for the spaces $\left.\mathcal{M}\left(g_{+}, \eta_{+}\right)\right)$.

Let us first stress the role played by some symmetries of a factorizable Lie group. The space $G \times \mathfrak{g}^{*}$ can be considered as a $G_{+} \times G_{-}$-space, if as above $G \times \mathfrak{g}^{*} \simeq T^{*} G$ via left trivialization and we lift the $G_{+} \times G_{-}$-action on $G$ given by

$$
G_{+} \times G_{-} \times G \rightarrow G:\left(a_{+}, a_{-} ; g\right) \mapsto a_{+} g a_{-}^{-1} .
$$


By using the facts that the action is lifted and the symplectic form on $G \times \mathfrak{g}^{*}$ is exact, we can determine the momentum map associated with this action; then we obtain that

$$
\begin{aligned}
J: G \times \mathfrak{g}^{*} & \rightarrow \mathfrak{g}_{-}^{\circ} \times \mathfrak{g}_{+}^{\circ} \\
(g, \xi) & \mapsto\left(\Pi_{\mathfrak{g}_{-}^{\circ}}\left(A d_{g}^{G} \xi\right), \Pi_{\mathfrak{g}_{+}^{\circ}}(\xi)\right),
\end{aligned}
$$

where $A d^{G}$ indicates the coadjoint action of $G$ on $\mathfrak{g}^{*}$. Let us now define the submanifold

$$
\Lambda_{\eta_{+} \eta_{-}}:=\left\{(g, \xi) \in G \times \mathfrak{g}^{*} / \Pi_{\mathfrak{g}_{-}^{\circ}}\left(A d_{g}^{G} \xi\right)=\eta_{+}, \Pi_{\mathfrak{g}_{+}^{\circ}}(\xi)=\eta_{-}\right\},
$$

for each pair $\eta_{+} \in \mathfrak{g}_{-}^{\circ}, \eta_{-} \in \mathfrak{g}_{+}^{\circ}$. We have the following lemma.

Lemma: Let $\xi_{ \pm} \in \mathfrak{g}_{\mp}^{\circ}, a_{+} \in G_{+}, a_{-} \in G_{-}$be arbitrary elements. Then the formulas

$$
\begin{aligned}
& a_{+} \cdot \xi_{+}:=\Pi_{\mathfrak{g}_{-}^{\circ}}\left(A d_{a_{+}}^{G} \xi_{+}\right), \\
& a_{-} \cdot \xi_{-}:=\Pi_{\mathfrak{g}_{+}^{\circ}}\left(A d_{a_{-}}^{G} \xi_{-}\right),
\end{aligned}
$$

define an action of $G_{ \pm}$on $\mathfrak{g}_{\mp}^{\circ}$; in fact, under the identification $\mathfrak{g}_{ \pm}^{*} \simeq \mathfrak{g}_{\mp}^{\circ}$ induced by the decomposition $\mathfrak{g}=\mathfrak{g}_{+}^{\circ} \oplus \mathfrak{g}_{-}^{\circ}$,these actions are just the coadjoint actions of each factor on the dual of its Lie algebras.

Note: The symbol $\mathcal{O}_{\xi_{ \pm}}^{G_{ \pm}}$will denote the orbit in $\mathfrak{g}_{\mp}^{\circ}$ under the actions defined in the previous lemma. Additionally, for each $\xi \in \mathfrak{g}^{*}$, the form $\xi^{b} \in \mathfrak{g}^{*}$ is given by $\xi^{b}:=B(\xi, \cdot)$, where $B(\cdot, \cdot)$ is the invariant bilinear form on $\mathfrak{g}^{*}$ induced by the Killing form.

Therefore $\Lambda_{\eta_{+} \eta_{-}}=J^{-1}\left(\eta_{+}, \eta_{-}\right)$and, taking into account the Marsden-Weinstein reduction (see Ref. 2), the projection of $\Lambda_{\eta_{+} \eta_{-}}$on $\Lambda_{\eta_{+} \eta_{-}} /\left(G_{+}\right)_{\eta_{+}} \times\left(G_{-}\right)_{\eta_{-}}$is presymplectic, and the solution curves for the dynamical system defined there by the invariant Hamiltonian $\mathcal{H}(g, \xi):=\frac{1}{2} \xi\left(\xi^{b}\right)$ are closely related with the solution curves of the system induced in the quotient. To work out these equations, let us introduce some convenient coordinates. The map $L_{\eta_{+} \eta_{-}}: \Lambda_{\eta_{+} \eta_{-}} \longrightarrow \mathcal{O}_{\eta_{+}}^{G_{+}} \times \mathcal{O}_{\eta_{-}}^{G_{-}}$ defined as

$$
L_{\eta_{+} \eta_{-}}(g, \xi)=\left(\Pi_{\mathfrak{g}_{-}^{\circ}} A d_{g_{+}^{-1}}^{G} \eta_{+}, \Pi_{\mathfrak{g}_{+}^{\circ}} A d_{g_{-}}^{G} \eta_{-}\right)=\left(\Pi_{\mathfrak{g}_{-}^{\circ}} A d_{g_{-}}^{G} \xi, \Pi_{\mathfrak{g}_{+}^{\circ}} A d_{g_{-}}^{G} \xi\right),
$$

where $g=g_{+} g_{-}$, induces a diffeomorphism on $\Lambda_{\eta_{+} \eta_{-}} /\left[\left(G_{+}\right)_{\eta_{+}} \times\left(G_{-}\right)_{\eta_{-}}\right]$. If $(g, \xi ; X, \lambda)$ is a tangent vector to $G \times \mathfrak{g}^{*}$ at ( $g, \xi$ ) (all the relevant bundles are left trivialized) then the derivative of $L_{\eta_{+} \eta_{-}}$is

$$
\begin{aligned}
& \left.\left(L_{\eta_{+} \eta_{-}}\right)_{*}\right|_{(g, \xi)}(g, \xi ; X, \lambda) \\
= & \left(-\Pi_{\mathfrak{g}_{-}} a d_{X_{+}}^{\mathfrak{g}} A d_{g_{+}^{-1}}^{G} \eta_{+}, \Pi_{\mathfrak{g}_{+}^{\circ}} a d_{X_{-}}^{\mathfrak{g}} A d_{g_{-}}^{G} \eta_{-}\right) \\
= & \left(\Pi_{\mathfrak{g}_{-}^{\circ}} a d_{X_{-}}^{\mathfrak{g}} A d_{g_{-}}^{G} \xi+\Pi_{\mathfrak{g}_{-}^{\circ}} A d_{g_{-}}^{G} \lambda, \Pi_{\mathfrak{g}_{+}^{\circ}} a d_{X_{-}}^{\mathfrak{g}} A d_{g_{-}}^{G} \xi+\Pi_{\mathfrak{g}_{+}^{\circ}} A d_{g_{-}}^{G} \lambda\right),
\end{aligned}
$$

if and only if $g=g_{+} g_{-}, X_{ \pm}=\Pi_{\mathfrak{g}_{ \pm}}\left(A d_{g_{-}}^{G} X\right)$. So the following remarkable result is true.

Proposition: Let $\mathcal{O}_{\eta_{+}}^{G_{+}} \times \mathcal{O}_{\eta_{-}}^{G_{-}}$be the phase space whose symplectic structure is $\omega_{\eta_{+} \eta_{-}}=\omega_{\eta_{+}}-$ $\omega_{\eta_{-}}$, where $\omega_{\eta_{ \pm}}$are the corresponding Kirillov-Kostant symplectic structures on each orbit. If $i_{\eta_{+} \eta_{-}}: \Lambda_{\eta_{+} \eta_{-}} \hookrightarrow G \times \mathfrak{g}^{*}$ is the inclusion map, then we have

$$
i_{\eta_{+} \eta_{-}}^{*} \omega=L_{\eta_{+} \eta_{-}}^{*} \omega_{\eta_{+} \eta_{-}} .
$$

Proof: $\operatorname{Let}\left(\varsigma_{+}, \varsigma_{-}\right)=\left(\Pi_{\mathfrak{g}_{-}^{\circ}}\left(A d_{a_{+}}^{G} \eta_{+}\right), \Pi_{\mathfrak{g}_{+}^{\circ}}\left(A d_{a_{-}}^{G} \eta_{-}\right)\right)$be an arbitrary element of $\mathcal{O}_{\eta_{+}}^{G_{+}} \times \mathcal{O}_{\eta_{-}}^{G_{-}}$, then the tangent space at this point is given by

$$
T_{\left(\varsigma_{+}, \zeta_{-}\right)}\left(\mathcal{O}_{\eta_{+}}^{G_{+}} \times \mathcal{O}_{\eta_{-}}^{G_{-}}\right)=\left\{\left(\Pi_{\mathfrak{g}_{-}^{\circ}} a d_{X_{+}}^{\mathfrak{g}} \varsigma_{+}, \Pi_{\mathfrak{g}_{+}^{\circ}} a d_{X_{-}}^{\mathfrak{g}} \varsigma_{-}\right) / X_{ \pm} \in \mathfrak{g}_{ \pm}\right\} .
$$


The symplectic structure $\omega_{\eta_{+} \eta_{-}}$is given in these terms as

$$
\begin{aligned}
& \left\langle\omega_{\eta_{+} \eta_{-}},\left(\Pi_{\mathfrak{g}_{-}^{\circ}} a d_{X_{+}}^{\mathfrak{g}} \varsigma_{+}, \Pi_{\mathfrak{g}_{+}^{\circ}} a d_{X_{-}}^{\mathfrak{g}} \varsigma_{-}\right) \otimes\left(\Pi_{\mathfrak{g}_{-}^{\circ}} a d_{Y_{+}}^{\mathfrak{g}} \varsigma_{+}, \Pi_{\mathfrak{g}_{+}^{\circ}} a d_{Y_{-}}^{\mathfrak{g}} \varsigma_{-}\right)\right\rangle_{\left(\varsigma_{+}, \varsigma_{-}\right)} \\
= & \left\langle\varsigma_{+},\left[X_{+}, Y_{+}\right]\right\rangle-\left\langle\varsigma_{-},\left[X_{-}, Y_{-}\right]\right\rangle .
\end{aligned}
$$

Let us take now an element $(g, \xi ; X, \lambda)$ tangent to $\Lambda_{\eta_{+} \eta_{-}}$at $(g, \xi)$; then it is true that

$$
\left\{\begin{array}{l}
\Pi_{\mathfrak{g}_{-}^{\circ}}\left(A d_{g}^{G}\left(a d_{X}^{\mathfrak{g}} \xi+\lambda\right)\right)=0 \\
\Pi_{\mathfrak{g}_{+}^{\circ}}(\lambda)=0 .
\end{array}\right.
$$

Because of $g=g_{+} g_{-}$, the first condition can be written as

$$
\Pi_{\mathfrak{g}_{-}^{\circ}}\left(A d_{g_{+}}^{G}\left(a d_{A d_{g_{-}} X}^{\mathfrak{g}} A d_{g_{-}}^{G} \xi+A d_{g_{-}}^{G} \lambda\right)\right)=0
$$

and because of the nondegeneracy condition (that is, such that $A d_{g}^{G} \mathfrak{g}_{+}^{\circ} \cap \mathfrak{g}_{-}^{\circ}=0$ for all $g \in G$,), it is equivalent to

$$
\Pi_{\mathfrak{g}_{-}^{\circ}}\left(a d_{A d_{-} X}^{\mathfrak{g}} A d_{g_{-}}^{G} \xi+A d_{g_{-}}^{G} \lambda\right)=0 .
$$

Let $(g, \xi ; X, \lambda),(g, \xi ; Y, \mu) \in T_{(g, \xi)} \Lambda_{\eta_{+} \eta_{-}}$; then by evaluating on the canonical form we have that

$$
\begin{aligned}
& \langle\omega,(g, \xi ; X, \lambda) \otimes(g, \xi ; Y, \mu)\rangle_{(g, \xi)} \\
= & \langle\lambda, Y\rangle-\langle\mu, X\rangle-\langle\xi,[X, Y]\rangle \\
= & \left\langle\Pi_{\mathfrak{g}_{-}^{\circ}} A d_{g_{-}}^{G} \lambda, Y_{+}\right\rangle+\left\langle\Pi_{\mathfrak{g}_{+}^{\circ}} A d_{g_{-}}^{G} \lambda, Y_{-}\right\rangle \\
& -\left\langle\Pi_{\mathfrak{g}_{-}^{\circ}} A d_{g_{-}}^{G} \mu, X_{+}\right\rangle-\left\langle\Pi_{\mathfrak{g}_{+}^{\circ}} A d_{g_{-}}^{G} \mu, X_{+}\right\rangle \\
& -\left\langle A d_{g_{-}}^{G} \xi,\left[X, Y_{+}\right]\right\rangle-\left\langle A d_{g_{-}}^{G} \xi,\left[X_{-}, Y\right]\right\rangle-\left\langle A d_{g_{-}}^{G} \xi,\left[X_{+}, Y_{-}\right]\right\rangle \\
= & \left\langle\Pi_{\mathfrak{g}_{-}^{\circ}}\left(A d_{g_{-}}^{G} \lambda+a d_{X}^{\mathfrak{g}} A d_{g_{-}}^{G} \xi\right), Y_{+}\right\rangle+\left\langle\Pi_{\mathfrak{g}_{+}^{\circ}} A d_{g_{-}}^{G} \lambda, Y_{-}\right\rangle \\
& -\left\langle\Pi_{\mathfrak{g}_{-}^{\circ}}\left(A d_{g_{-}}^{G} \mu+a d_{Y_{-}}^{\mathfrak{g}} A d_{g_{-}}^{G} \xi\right), X_{+}\right\rangle-\left\langle\Pi_{\mathfrak{g}_{+}^{\circ}} A d_{g_{-}}^{G} \mu, X_{+}\right\rangle \\
& -\left\langle A d_{g_{-}}^{G} \xi,\left[X X_{-}, Y_{-}\right]\right\rangle,
\end{aligned}
$$

where we have used the notation according to which $(X)_{ \pm}=\Pi_{\mathfrak{g}_{ \pm}}\left(A d_{g_{-}}^{G} X\right)$ and $(Y)_{ \pm}$ $=\Pi_{\mathfrak{g}_{ \pm}}\left(A d_{g_{-}}^{G} Y\right)$. The first term in this expression annihilates because of Eq. (37); additionally, the second and fourth terms vanish as a consequence of the second equation in (36), which implies that $\lambda, \mu \in \mathfrak{g}_{-}^{\circ}$, and because this subspace is invariant for the $G_{-}$-action through the coadjoint action. Moreover, for the third term in the second hand side, we use Eq. (36) again, and therefore we can write

$$
\Pi_{\mathfrak{g}_{-}^{\circ}}\left(A d_{g_{-}}^{G} \mu+a d_{Y_{-}}^{\mathfrak{g}} A d_{g_{-}}^{G} \xi\right)\left(X_{+}\right)=-\left\langle A d_{g_{-}}^{G} \xi,\left[X_{+}, Y_{+}\right]\right\rangle
$$

and so

$$
\begin{aligned}
& \langle\omega,(g, \xi ; X, \lambda) \otimes(g, \xi ; Y, \mu)\rangle_{(g, \xi)} \\
= & \left\langle\Pi_{\mathfrak{g}_{-}^{\circ}} A d_{g_{-}}^{G} \xi,\left[X_{+}, Y_{+}\right]\right\rangle-\left\langle\Pi_{\mathfrak{g}_{+}^{\circ}} A d_{g_{-}}^{G} \xi,\left[X_{-}, Y_{-}\right]\right\rangle .
\end{aligned}
$$

From the symplectic structure on product of orbits and by using the expression for the derivative along the map $L_{\eta_{+} \eta_{-}}$, we conclude the proof.

Let us now address the dynamical data related to the AKS system. We have shown that the symplectic manifold $\left(\mathcal{O}_{\eta_{+}}^{G_{+}} \times \mathcal{O}_{\eta_{-}}^{G_{-}}, \omega_{\eta_{+} \eta_{-}}\right)$is symplectomorphic to the reduced space associated with the $G_{+} \times G_{-}$-action defined above on $G \times \mathfrak{g}^{*}$. As it was pointed out above, the Hamilton function $\mathcal{H}(g, \xi)=\frac{1}{2}\left\langle\xi, \xi^{b}\right\rangle$ is invariant for this action, and it implies that the solutions of the 
dynamical system defined by such a Hamiltonian on $G \times \mathfrak{g}^{*}$ are in one-to-one correspondence with those of the dynamical system induced on $\mathcal{O}_{\eta_{+}}^{G_{+}} \times \mathcal{O}_{\eta_{-}}^{G_{-}}$by the Hamiltonian $\mathcal{H}_{\eta_{+} \eta_{-}}$(Ref. 2) defined according to the formula

$$
i_{\eta_{+} \eta_{-}}^{*} \mathcal{H}=L_{\eta_{+} \eta_{-}}^{*} \mathcal{H}_{\eta_{+} \eta_{-}} .
$$

Let us note now that if $L_{\eta_{+} \eta_{-}}(g, \xi)=\left(\varsigma_{+}, \varsigma_{-}\right)$, then $A d_{g_{-}}^{G} \xi=\varsigma_{+}+\varsigma_{-}$, and so

$$
\begin{aligned}
\mathcal{H}_{\eta_{+} \eta_{-}}\left(\varsigma_{+}, \varsigma_{-}\right) & =\frac{1}{2}\left\langle A d_{g_{-}^{-1}}^{G}\left(\varsigma_{+}+\varsigma_{-}\right),\left(A d_{g_{-}^{-1}}^{G}\left(\varsigma_{+}+\varsigma_{-}\right)\right)^{b}\right\rangle \\
& =\frac{1}{2}\left\langle A d_{g_{-}^{-1}}^{G}\left(\varsigma_{+}+\varsigma_{-}\right), A d_{g_{-}^{-1}}\left(\varsigma_{+}+\varsigma_{-}\right)^{b}\right\rangle \\
& =\frac{1}{2}\left\langle\varsigma_{+}, \varsigma_{+}^{b}\right\rangle+\frac{1}{2}\left\langle\varsigma_{-}, \varsigma_{-}^{b}\right\rangle+\left\langle\varsigma_{+}, \varsigma_{-}^{b}\right\rangle .
\end{aligned}
$$

Therefore,

$$
\begin{aligned}
& \left\langle d \mathcal{H}_{\eta_{+} \eta_{-}},\left.\left(\Pi_{\mathfrak{g}_{-}^{\circ}} a d_{X}^{\mathfrak{g}} \varsigma_{+}, \Pi_{\mathfrak{g}_{+}^{\circ}} a d_{Y}^{\mathfrak{g}} \varsigma_{-}\right)\right|_{\left(\zeta_{+}, \varsigma_{-}\right)}\right. \\
= & \left\langle\Pi_{\mathfrak{g}_{-}^{\circ}} a d_{X}^{\mathfrak{g}} \varsigma_{+}, \varsigma_{+}^{\mathrm{b}}+\varsigma_{-}^{\mathrm{b}}\right\rangle+\left\langle\Pi_{\mathfrak{g}_{+}^{\circ}} a d_{Y}^{\mathfrak{g}} \varsigma_{-}, \varsigma_{+}^{\mathrm{b}}+\varsigma_{-}^{\mathrm{b}}\right\rangle
\end{aligned}
$$

and the Hamiltonian vector field is then

$$
\left.V_{\mathcal{H}_{\eta_{+} \eta_{-}}}\right|_{\left(\varsigma_{+}, \varsigma_{-}\right)}=\left(\Pi_{\mathfrak{g}_{-}^{\circ}} a d_{\Pi_{\mathfrak{g}_{+}}\left(s_{+}^{\mathrm{b}}+\varsigma_{-}^{\mathrm{b}}\right)}^{\mathfrak{g}} \varsigma_{+}, \Pi_{\mathfrak{g}_{+}^{\circ}} a d_{\Pi_{\mathfrak{g}_{-}}\left(s_{+}^{\mathrm{b}}+\varsigma_{-}^{\mathrm{b}}\right)}^{\mathfrak{g}} \varsigma_{-}\right) .
$$

In general, in dealing with a dynamical systems via reduction it is expected the reduced system to be easier to solve than the original because it involves less degrees of freedom, and the solution of the original system is found by lifting the solution of the reduced system. In case of the AKS systems, we proceed in the reverse direction: to this end let us note that the dynamical system on $G \times \mathfrak{g}^{*}$ determined by $\mathcal{H}$ has Hamiltonian vector field given by

$$
\left.V_{\mathcal{H}}\right|_{(g, \xi)}=\left(\xi^{b},-a d_{\xi^{b}}^{\mathfrak{g}} \xi\right)=\left(\xi^{b}, 0\right)
$$

because of the invariance condition $a d_{X}^{\mathfrak{g}} X^{b}=0$ for all $X \in \mathfrak{g}$. Then the solution in the original phase space passing through $(g, \xi)$ at the initial time is

$$
t \mapsto\left(g \exp t \xi^{\mathrm{b}}, \xi\right),
$$

and if this initial data verify $\Pi_{\mathfrak{g}_{-}^{\circ}}\left(A d_{g}^{\sharp} \xi\right)=\eta_{+}, \Pi_{\mathfrak{g}_{+}^{\circ}}(\xi)=\eta_{-}$, then this curve belongs to $\Lambda_{\eta_{+} \eta_{-}}$for all $t$. Therefore the map,

$$
t \mapsto\left(\Pi_{\mathfrak{g}_{-}^{\circ}} A d_{\left(g_{+}(t)\right)^{-1}}^{G} \eta_{+}, \Pi_{\mathfrak{g}_{+}^{\circ}} A d_{g_{-}(t)}^{G} \eta_{-}\right),
$$

where $g_{ \pm}: \mathbb{R} \rightarrow G_{ \pm}$are the curves defined by the factorization problem $g_{+}(t) g_{-}(t)=g \exp t \xi^{b}$, is a solution for the dynamical system associated with the vector field (38), yielding to a differential equation which is harder to solve than the original system.

\section{AKS dynamics on the spaces $\mathcal{N}\left(g_{-}, \eta_{-}\right)$}

Let $\eta_{-} \in \mathfrak{g}_{+}^{\circ}$ be a character. As it was shown above, the spaces $\mathcal{N}\left(g_{-}, \eta_{-}\right)$are $G$-spaces, and in particular $G_{+}$-spaces; therefore, there exists a momentum map $J_{+}: \mathcal{N}\left(g_{-}, \eta_{-}\right) \rightarrow \mathfrak{g}_{-}^{\circ}$. The connection with the dynamics on the phase spaces $\mathcal{N}\left(g_{-}, \eta_{-}\right)$is given by the following theorem, which is a consequence of the Hamiltonian reduction by stages. ${ }^{8}$

Theorem: Let $\eta_{+} \in \mathfrak{g}_{-}^{\circ}$ be an arbitrary element. The reduced space,

$$
J_{+}^{-1}\left(\eta_{+}\right) /\left(G_{+}\right)_{\eta_{+}},
$$

is therefore symplectomorphic to the orbit space $\mathcal{O}_{\eta_{+}}^{G_{+}} \times \mathcal{O}_{\eta_{-}}^{G_{-}}$. 
Proof: Simply note that $G_{+}$and $G_{-}$commutes each other as subgroups in $G_{+} \times G_{-}$, and the same is true for their actions on $G$; then by performing the reduction into $J^{-1}\left(\eta_{+}, \eta_{-}\right) /\left[\left(G_{+}\right)_{\eta_{+}} \times\left(G_{-}\right)_{\eta_{-}}\right]$by stages, first using the right $G_{-}$-action and then the left $G_{+^{-}}$ action, we prove the theorem.

The following diagram can be useful to explain the contents of the previous statement:

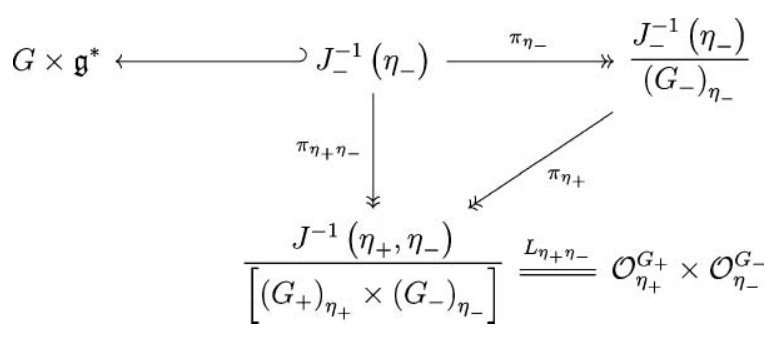

where the $\pi$-maps denote the canonical projections onto the corresponding quotients, here $M=$ $G / G_{-}$. Accordingly, the picture can be described as follows: the Hamiltonian $\mathcal{H}(g, \xi)=\frac{1}{2} \xi\left(\xi^{b}\right)$ defines on $G \times \mathfrak{g}^{*}$ a dynamical system whose solutions are easier to be found; they are given by the formula

$$
t \mapsto\left(g \exp t \xi^{b}, \xi\right)
$$

for any initial data $\left(g_{\circ}, \xi_{\circ}\right) \in G \times \mathfrak{g}^{*}$. Since $G$ is a factorizable Lie group, $G=G_{+} G_{-}$for some subgroups $G_{ \pm} \subset G$, such that $G_{+} \cap G_{-}=\{e\}$; on $G$ there exists a $G_{+} \times G_{-}$-action defined as

$$
\left(a_{+}, a_{-}\right) \cdot g:=a_{+} g a_{-}^{-1}
$$

for all $a_{+} \in G_{+}, a_{-} \in G_{-}$, and $g \in G$. This action lifts to a symplectic action on $G \times \mathfrak{g}^{*}$ (left trivialized), and $\mathcal{H}$ turns out to be an invariant Hamiltonian; therefore there exists a Hamiltonian system on the reduced space with solutions related to the solutions of the system determined by $\mathcal{H}$ on $G \times \mathfrak{g}^{*}$. Because of the discussion in the previous subsection, the dynamical system so induced on $J^{-1}\left(\eta_{+}, \eta_{-}\right) /\left[\left(G_{+}\right)_{\eta_{+}} \times\left(G_{-}\right)_{\eta_{-}}\right]$is the AKS system.

Let us now suppose that $\eta_{-} \in \mathfrak{g}_{+}^{\circ}$ is a character for the coadjoint action. It was shown above that the spaces $\mathcal{N}\left(g_{-}, \eta_{-}\right)$are slices for the right $G_{-}$-action on $G \times\left(\mathfrak{g}_{-}^{\circ}+\eta_{-}\right)$, and therefore they are symplectic submanifolds of $G \times \mathfrak{g}^{*}$. Further, it was shown that these spaces are symplectomorphic to the space $G \times{ }_{G_{-}}\left(\mathfrak{g}_{-}^{\circ}+\eta_{-}\right)$, the reduced space for the right $G_{-}$-action on $G \times \mathfrak{g}^{*}$. Additionally, this reduced space is a $G$-space via the lift of the canonical action of $G$ on its homogeneous space $M=G / G_{-}$; in particular, it is a $G_{+}$-space, and we can apply the reduction scheme once more. The reduction by stages theorem guarantees that the resulting space is symplectomorphic to the AKS system as obtained in Sec. IV C, and the solution curves for the system induced on $G \times_{G_{-}}\left(\mathfrak{g}_{-}^{\circ}+\eta_{-}\right)$by $\mathcal{H}$ projects down onto the solution curves for the AKS systems; we can think of them as an intermediate lift of the dynamics of the AKS system.

Let us now analyze the lifting of the dynamics to $\mathcal{N}\left(g_{-}, \eta_{-}\right)$. As before, here $\eta_{-}$is a character for the $A d^{*}$-action and $g_{-}$an arbitrary element in $G_{-}$. We want to describe the dynamical system on $\mathcal{N}\left(g_{-}, \eta_{-}\right)$defined through the reduction procedure described above. In order to achieve this, let us recall that the map

$$
\tilde{\pi}_{\eta_{-}}:=\pi_{\eta_{-}} \mid \mathcal{N}\left(g_{-}, \eta_{-}\right): \mathcal{N}\left(g_{-}, \eta_{-}\right) \rightarrow J_{-}^{-1}\left(\eta_{-}\right) / G_{-}=: M_{\eta_{-}}^{-},
$$

between this symplectic submanifold and the reduced space $M_{\eta_{-}}^{-}$is a symplectomorphism (i.e., the note at the end of Sec. III A 3). Let us denote by $\omega_{\mathcal{N}}$ the symplectic structure on $\mathcal{N}\left(g_{-}, \eta_{-}\right)$; now the reduction procedure defines on $M_{\eta_{-}}^{-}$both the symplectic structure $\omega_{\eta_{-}}$and the Hamiltonian function 
$\mathcal{H}_{\eta_{-}}$through the formulas

$$
\begin{aligned}
& \pi_{\eta_{-}}^{*} \omega_{\eta_{-}}=\omega_{\circ} \mid J_{-}^{-1}\left(\eta_{-}\right), \\
& \pi_{\eta_{-}}^{*} H_{\eta_{-}}=\mathcal{H} \mid J_{-}^{-1}\left(\eta_{-}\right),
\end{aligned}
$$

so that if $i_{\eta_{-}}: \mathcal{N}\left(g_{-}, \eta_{-}\right) \hookrightarrow G \times \mathfrak{g}^{*}$ is the inclusion, then

$$
\begin{aligned}
i_{\eta_{-}}^{*} \omega_{\circ} & =i_{\eta_{-}}^{*}\left[\omega_{\circ} \mid J_{-}^{-1}\left(\eta_{-}\right)\right] \\
& =i_{\eta_{-}}^{*} \pi_{\eta_{-}}^{*} \omega_{\eta_{-}} \\
& =\left(\pi_{\eta_{-}} \circ i_{\eta_{-}}\right)^{*} \omega_{\eta_{-}} \\
& =\tilde{\pi}_{\eta_{-}}^{*} \omega_{\eta_{-}} \\
& =\omega_{\mathcal{N}},
\end{aligned}
$$

and accordingly $\mathcal{H}_{\mathcal{N}}:=i_{\eta_{-}}^{*} \mathcal{H}$ verifies $\tilde{\pi}_{\eta_{-}}^{*} \mathcal{H}_{\eta_{-}}=\mathcal{H}_{\mathcal{N}}$. Therefore, the dynamics defined on $\mathcal{N}\left(g_{-}, \eta_{-}\right)$via the identification with the reduced space $M_{\eta_{-}}^{-}$is equivalent to the dynamics defined on the same space through the restriction of the dynamical data $\left(\omega_{0}, \mathcal{H}\right)$. These considerations enable to lift the AKS dynamics from the full reduced space $J^{-1}\left(\eta_{+}, \eta_{-}\right) /\left[\left(G_{+}\right)_{\eta_{+}} \times G_{-}\right]$to $M_{\eta_{-}}^{-}$; that is, if $(h, \xi)$ is an arbitrary point at $J_{-}^{-1}\left(\eta_{-}\right)$, then the solution $\gamma: t \mapsto\left(h \exp t \xi^{b}, \xi\right)$ for the dynamical system defined on $G \times \mathfrak{g}^{*}$ by the data $\left(\omega_{\circ}, \mathcal{H}\right)$ remains there for all $t$, and $t \mapsto \pi_{\eta_{-}}(\gamma(t))$ is the solution for the dynamical system defined on $M_{\eta_{-}}^{-}$via $\left(\omega_{\eta_{-}}, \mathcal{H}_{\eta_{-}}\right)$passing through $\pi_{\eta_{-}}(h, \xi)$. Finally, $t \mapsto\left[\tilde{\pi}_{\eta_{-}}^{-1} \circ \pi_{\eta_{-}}\right](\gamma(t))$ is the solution to the Hamilton equations on $\mathcal{N}\left(g_{-}, \eta_{-}\right)$associated with the dynamical data $\left(\omega_{\mathcal{N}}, \mathcal{H}_{\mathcal{N}}\right)$, with initial data $\gamma(0)=\tilde{\pi}_{\eta_{-}}^{-1} \circ \pi_{\eta_{-}}(h, \xi)$. An explicit formula for this solution curve can be given, because

$$
\begin{aligned}
{\left[\left(\tilde{\pi}_{\eta_{-}}\right)^{-1} \circ \pi_{\eta_{-}}\right]\left(h_{+} h_{-}, \xi_{+}+\eta_{-}\right) } & =\tilde{\pi}_{\eta_{-}}^{-1}\left(\left[h_{+} h_{-}, \xi_{+}+\eta_{-}\right]\right) \\
& =\tilde{\pi}_{\eta_{-}}^{-1}\left(\left[h_{+} g_{-}, A d_{g_{-}^{-1} h_{-}}^{G} \xi_{+}+\eta_{-}\right]\right) \\
& =\left(h_{+} g_{-}, A d_{g_{-}^{-1} h_{-}}^{G} \xi_{+}+\eta_{-}\right) .
\end{aligned}
$$

This means that the solution curve has the following form:

$$
t \mapsto\left(h_{+}(t) g_{-}, A d_{g_{-}^{-1} h_{-}(t)}^{G} \xi_{+}+\eta_{-}\right),
$$

where $h_{ \pm}: \mathbb{R} \rightarrow G_{ \pm}$are the solutions of the factorization problem $h_{+}(t) h_{-}(t)=h \exp t \xi^{b}$.

In the same vein, the previous setting can be applied in order to find the Hamiltonian vector field associated with the Hamiltonian $\mathcal{H}_{\mathcal{N}}$ on $\mathcal{N}\left(g_{-}, \eta_{-}\right)$. First of all, we need the following proposition.

Proposition: By left trivializing $T_{(h, \xi)}\left(G \times \mathfrak{g}^{*}\right)=T_{h} G \times T_{\xi} \mathfrak{g}^{*}=\mathfrak{g} \times \mathfrak{g}^{*}$, the derivative of the map $\Xi_{\eta_{-}}:=\tilde{\pi}_{\eta_{-}}^{-1} \circ \pi_{\nu}$ is given by

$$
\begin{aligned}
& \left.\Xi_{\eta_{-} *}\right|_{(h, \xi)}\left(X, \lambda_{+}\right) \\
= & \left(A d_{g_{-}^{-1}} \Pi_{\mathfrak{g}_{+}} A d_{h_{-}}^{G} X, A d_{g_{-}^{-1}}^{G} a d_{A d_{h_{-}} X_{-}}^{\mathfrak{g}} A d_{h_{-}}^{G} \xi_{+}+A d_{g_{-}^{-1} h_{-}}^{G} \lambda_{+}\right)
\end{aligned}
$$

for all $\left(X, \lambda_{+}\right) \in T_{(h, \xi)} J_{-}^{-1}\left(\eta_{-}\right) \subset \mathfrak{g} \times \mathfrak{g}^{*}$.

Proof: Let $h_{ \pm}: \mathbb{R} \rightarrow G_{ \pm}$be the solution curves for the factorization problem

$$
h_{+}(t) h_{-}(t)=h \exp t X
$$

then if

$$
X_{ \pm}:=L_{g_{ \pm}^{-1} *}\left\{\left.\frac{d}{d t}\right|_{t=0}\left[h_{ \pm}(t)\right]\right\}
$$


we see that

$$
X=A d_{h_{-}^{-1}}^{G} X_{+}+X_{-} .
$$

It is immediate from here to conclude that

$$
X_{+}=\Pi_{\mathfrak{g}_{+}}\left(A d_{h_{-}}^{G} X\right)
$$

and additionally

$$
A d_{h_{-}} X_{-}=\Pi_{\mathfrak{g}_{-}} A d_{h_{-}}^{G} X .
$$

By using the previous formula for map $\pi_{\eta_{-}}$and the identity

$$
\left.\frac{d}{d t}\right|_{t=0}\left[A d_{h_{-}(t)}^{G} \xi_{+}\right]=a d_{A d_{h_{-}} X_{-}}^{\mathfrak{g}} A d_{h_{-}}^{G} \xi_{+},
$$

we obtain the proposition.

Finally, it remains to take into account that

$$
\left.V_{\mathcal{H}_{\mathcal{N}}}\right|_{\left(g_{+} g_{-}, \eta_{+}+\eta_{-}\right)}=\left.\Xi_{\eta_{-} *}\right|_{(h, \xi)}\left(\left.V_{\mathcal{H}}\right|_{(h, \xi)}\right)
$$

for any $(h, \xi) \in \Xi_{\eta_{-}}^{-1}\left(g_{+} g_{-}, \eta_{+}+\eta_{-}\right)$. From the expression

$$
\left.V_{\mathcal{H}}\right|_{(h, \xi)}=\left(\xi^{b}, 0\right)
$$

in the left trivialization, and by realizing that $(h, \xi) \in \Xi_{\eta_{-}}^{-1}\left(g_{+} g_{-}, \eta_{+}+\eta_{-}\right)$if and only if

$$
\left\{\begin{array}{l}
h_{+}=g_{+} \\
\xi_{+}=A d_{h_{-}^{-1} g_{-}}^{G} \eta_{+}
\end{array}\right.
$$

we can conclude that

$$
\left.V_{H_{\mathcal{N}}}\right|_{(g, \eta)}=\left(A d_{g_{-}^{-1}}^{G} \Pi_{\mathfrak{g}_{+}} A d_{g_{-}} \eta_{+}^{\mathrm{b}}, A d_{g_{-}^{-1}}^{G} a d_{\Pi_{\mathfrak{g}_{-}} A d_{g_{-}} \eta_{+}^{b}}^{\mathfrak{g}} A d_{g_{-}}^{G} \eta_{+}\right)
$$

for $(g, \eta)=\left(g_{+} g_{-}, \eta_{+}+\eta_{-}\right)$. It was used here that the $A d$-invariance of the Killing form implies the equivariance formula

$$
A d_{h_{-}^{-1}}^{G} \xi_{+}^{b}=\left(A d_{h_{-}}^{G} \xi_{+}\right)^{b}
$$

for all $g_{-} \in G_{-}, \xi_{+} \in \mathfrak{g}_{-}^{\circ}$.

It is interesting to relate this vector field with the vector field obtained in Eq. (30). If $\mathrm{h}(\xi)$ : $=\frac{1}{2}\left\langle\xi, \xi^{b}\right\rangle$, then $\mathcal{L}_{\mathrm{h}}(\eta)=\eta^{\mathrm{b}}$ and the vector field (30) (evaluated at $\left(h_{+} h_{-}, \eta_{+}+\eta_{-}\right)$) can be written as

$$
\begin{aligned}
& V_{\mathrm{h} \circ \Phi^{L}}^{D} \\
= & \left(\begin{array}{c}
g A d_{h_{-}^{-1}} \Pi_{\mathfrak{g}_{+}^{\circ}} A d_{h_{-}}\left(\eta_{+}+\eta_{-}\right)^{\mathrm{b}}, \\
A d_{h_{-}^{-1}} \Pi_{\mathfrak{g}_{-}^{\circ}} A d_{h_{-}}\left[\eta_{+}+\eta_{-}, A d_{h_{-}^{-1}} \Pi_{\mathfrak{g}_{+}^{\circ}} A d_{h_{-}}\left(\eta_{+}+\eta_{-}\right)^{\mathrm{b}}\right]
\end{array}\right) \\
= & \left(g A d_{h_{-}^{-1}} \Pi_{\mathfrak{g}_{+}^{\circ}} A d_{h_{-}}\left(\eta_{+}\right), A d_{h_{-}^{-1}} \Pi_{\mathfrak{g}_{-}^{\circ}} A d_{h_{-}}\left[\eta_{+}, A d_{h_{-}^{-1}} \Pi_{\mathfrak{g}_{+}^{\circ}} A d_{h_{-}}\left(\eta_{+}\right)\right]\right),
\end{aligned}
$$

where it was used that, under the performed identifications, $\eta_{ \pm}^{b}=\eta_{ \pm}$for all $\eta_{ \pm} \in \mathfrak{g}_{ \pm}^{\circ}$, and moreover, that $\eta_{-} \in \mathfrak{g}_{-}^{\circ}$ is a character. Finally,

$$
\begin{aligned}
V_{\mathrm{h}_{\circ} \Phi^{L}}^{D} & =\left(g A d_{h_{-}^{-1}} \Pi_{\mathfrak{g}_{+}^{\circ}} A d_{h_{-}}\left(\eta_{+}\right), A d_{h_{-}^{-1}} \Pi_{\mathfrak{g}_{-}^{\circ}}\left[A d_{h_{-}} \eta_{+}, \Pi_{\mathfrak{g}_{+}^{\circ}} A d_{h_{-}}\left(\eta_{+}\right)\right]\right) \\
& =\left(g A d_{h_{-}^{-1}} \Pi_{\mathfrak{g}_{+}^{\circ}} A d_{h_{-}}\left(\eta_{+}\right),-A d_{h_{-}^{-1}} \Pi_{\mathfrak{g}_{-}^{\circ}}\left[A d_{h_{-}} \eta_{+}, \Pi_{\mathfrak{g}_{-}^{\circ}} A d_{h_{-}}\left(\eta_{+}\right)\right]\right) \\
& =\left(g A d_{h_{-}^{-1}} \Pi_{\mathfrak{g}_{+}^{\circ}} A d_{h_{-}}\left(\eta_{+}\right),-A d_{h_{-}^{-1}}\left[A d_{h_{-}} \eta_{+}, \Pi_{\mathfrak{g}_{-}^{\circ}} A d_{h_{-}}\left(\eta_{+}\right)\right]\right)
\end{aligned}
$$


gives us the desired expression, to be compared with Eq. (30).

\section{EXAMPLES}

\section{A. Semidirect product}

Let $G_{-}=V$, a representation space of $G_{+}$regarded as the Abelian group underlying the vector space, so that $G=G_{+} \ltimes V$. In this case, we consider the semidirect product structure - : $G \times G \longrightarrow G$ defined as

$$
(a, u) \bullet(b, v)=\left(a b, b^{-1} u+v\right),
$$

where $b^{-1} u$ stands for the left action of $G_{+}$on $V$. This is in fact an associative product. The left and right translation in this structure are

$$
\begin{aligned}
& \left(R_{(b, v)}^{\bullet}\right)_{*(a, u)}(V, z)=\left(\left(R_{b}\right)_{*} V, b^{-1} z\right)_{\left(a b, b^{-1} u+v\right)}, \\
& \left(L_{(b, v)}^{\bullet}\right)_{*(a, u)}(V, z)=\left(\left(L_{b}\right)_{*} V,-\left(a^{-1} V\right)\left(a^{-1} v\right)+z\right)_{\left(b a, a^{-1} v+u\right)},
\end{aligned}
$$

and we may now calculate the adjoint action on the Lie algebra of $G$, having in mind that the inverse of $(a, v)$ is now $\left(a^{-1},-a v\right)$,

$$
A d_{(b, v)}^{\bullet}(X, z)=\left(A d_{b} X,-b X v+b z\right)
$$

and the infinitesimal adjoint action of $G_{+} \times V$ on $\mathfrak{g}_{+} \oplus V$,

$$
[(Y, v),(X, z)]_{\mathfrak{g}_{+} \oplus V}^{\bullet}=([Y, X], Y z-X v),
$$

defining the Lie algebra structure on the vector space $\mathfrak{g}_{+} \oplus V$, underlying the Lie group semidirect product structure of $G$.

Let us first to determine the Poisson bracket on the submanifolds defined by $\Psi: G \times \mathfrak{g}^{*} \longrightarrow$ $V \times V^{*}$, namely, $\mathcal{N}(u, \alpha)$ by applying the expression (20). So, we need the explicit expressions for the adjoint action of $(e, v) \in G$,

$$
\begin{gathered}
A d_{(e, v)}^{\bullet}(0, u)=\left(A d_{e} 0,-(e 0) v+e u\right)=u, \\
A d_{(e, v)}^{\bullet}(X, 0)=\left(A d_{e} X,-e X v+e 0\right)=(X,-X v),
\end{gathered}
$$

and its projections on the Lie algebra of $G_{+}$,

$$
\begin{aligned}
\Pi_{\mathfrak{g}_{+}} A d_{v}^{G} u & =\Pi_{\mathfrak{g}_{+}} u=0, \\
\Pi_{\mathfrak{g}_{+}} A d_{v}^{G} X & =\Pi_{\mathfrak{g}_{+}}(X,-X v)=X .
\end{aligned}
$$

Now, we are ready to get the Poisson-Dirac bracket

$$
\begin{aligned}
& \{\mathcal{F}, \mathcal{G}\}^{\mathcal{N}}\left(\left(g_{+}, u\right),\left(\eta_{+}, \alpha\right)\right) \\
= & \left\langle\mathbf{d} \mathcal{F},\left(g_{+}, u\right) \Pi_{\mathfrak{g}_{+}} \delta \mathcal{G}\right\rangle-\left\langle\mathbf{d} \mathcal{G},\left(g_{+}, u\right) \Pi_{\mathfrak{g}_{+}} \delta \mathcal{F}\right\rangle-\left\langle\eta_{+},\left[\Pi_{\mathfrak{g}_{+}} \delta \mathcal{F}, \Pi_{\mathfrak{g}_{+}} \delta \mathcal{G}\right]\right\rangle,
\end{aligned}
$$

where $\left(g_{+}, u\right) X$ stands for above defined left translation action on $\mathfrak{g}$, that in this case reduces to

$$
\left(L_{\left(g_{+}, u\right)}^{\bullet}\right)_{*(e, 0)}\left(\Pi_{\mathfrak{g}_{+}} \delta \mathcal{G}, 0\right)=\left(\left(L_{g_{+}}\right)_{*} \Pi_{\mathfrak{g}_{+}} \delta \mathcal{G},-\left(\Pi_{\mathfrak{g}_{+}} \delta \mathcal{G}\right) u\right)_{\left(g_{+}, u\right)} .
$$

Carrying this result to the Dirac bracket we get

$$
\begin{aligned}
& \{\mathcal{F}, \mathcal{G}\}^{\mathcal{N}}\left(\left(g_{+}, u\right),\left(\eta_{+}, \alpha\right)\right) \\
= & \left\langle\mathbf{d} \mathcal{F}, g_{+}\left(\Pi_{\mathfrak{g}_{+}} \delta \mathcal{G}\right)\right\rangle-\left\langle\mathbf{d} \mathcal{G}, g_{+}\left(\Pi_{\mathfrak{g}_{+}} \delta \mathcal{F}\right)\right\rangle-\left\langle\eta_{+},\left[\Pi_{\mathfrak{g}_{+}} \delta \mathcal{F}, \Pi_{\mathfrak{g}_{+}} \delta \mathcal{G}\right]\right\rangle .
\end{aligned}
$$


In particular, for $u=0$ and $\alpha=0$, one retrieves the usual result for the cotangent bundle of Lie groups. This result also applies when $V$ is the trivial representation space.

Let us analyze now the other family of symplectic submanifolds $\mathcal{M}\left(g_{+}, \eta_{+}\right)$defined by the fibration $\Upsilon: G \times \mathfrak{g}^{*} \longrightarrow G_{+} \times \mathfrak{g}_{+}^{*}$. The Dirac bracket in this case is

$$
\begin{aligned}
& \{\mathcal{F}, \mathcal{H}\}^{\mathcal{M}}\left(\left(g_{+}, u\right),\left(\eta_{+}, \alpha\right)\right) \\
= & \left\langle g \mathbf{d} \mathcal{F}, \Pi_{V} \delta \mathcal{H}\right\rangle-\left\langle g \mathbf{d} \mathcal{H}, \Pi_{V} \delta \mathcal{F}\right\rangle-\left\langle\alpha,\left[\Pi_{V} \delta \mathcal{F}, \Pi_{V} \delta \mathcal{H}\right]\right\rangle \\
= & \left\langle\mathbf{d} \mathcal{F},\left(L_{\left(g_{+}, u\right)}^{\cdot}\right)_{*(e, 0)} \Pi_{V} \delta \mathcal{H}\right\rangle-\left\langle\mathbf{d} \mathcal{H},\left(L_{\left(g_{+}, u\right)}^{\cdot}\right)_{*(e, 0)} \Pi_{V} \delta \mathcal{F}\right\rangle
\end{aligned}
$$

then, since

$$
\left(L_{(b, v)}^{\bullet}\right)_{*(a, u)}(0, z)=(0, z)_{\left(b a, a^{-1} v+u\right)},
$$

we have

$$
\{\mathcal{F}, \mathcal{H}\}^{\mathcal{M}}\left(\left(g_{+}, u\right),\left(\eta_{+}, \alpha\right)\right)=\left\langle\Pi_{V^{*}} \mathbf{d} \mathcal{F}, \Pi_{V} \delta \mathcal{H}\right\rangle-\left\langle\Pi_{V^{*}} \mathbf{d} \mathcal{H}, \Pi_{V} \delta \mathcal{F}\right\rangle
$$

that coincides with the canonical symplectic form on $V \times V^{*}$.

\section{B. $S L(2, \mathbb{C})=S U(2) \times B$}

Let us work out the case of $G=S L(2, \mathbb{C})$ and its Iwasawa decomposition $S L(2, \mathbb{C}) \cong S U(2)$ $\times B$, where $B$ is the group of $2 \times 2$ complex upper triangular matrices, with real diagonal and determinant equal to 1 , and we identify $G_{+}=S U(2)$ and $G_{-}=B$.

The Killing form for $\mathfrak{s l}_{2}(\mathbb{C})$ is $\kappa(X, Y):=\operatorname{tr}(\operatorname{ad}(X) \operatorname{ad}(Y))=4 \operatorname{tr}(X Y)$, the restrictions to $\mathfrak{s u}_{2}$, $\mathfrak{a}$, and $\mathfrak{n}$ are negative defined; positive defined, and 0 , respectively. We consider the nondegenerate symmetric bilinear form on $\mathfrak{s h}_{2}(\mathbb{C})$,

$$
(X, Y)_{\mathfrak{s} \mathfrak{l}_{2}}=-\frac{1}{4} \operatorname{Im} \kappa(X, Y),
$$

which turns $\mathfrak{b}$ and $\mathfrak{s u}_{2}$ into isotropic subspaces. Also, we take the basis

$$
T_{1}=\left[\begin{array}{ll}
0 & i \\
i & 0
\end{array}\right], \quad T_{2}=\left[\begin{array}{cc}
0 & 1 \\
-1 & 0
\end{array}\right], \quad T_{3}=\left[\begin{array}{cc}
i & 0 \\
0 & -i
\end{array}\right]
$$

for $\mathfrak{s u}_{2}$, and

$$
T^{1}=\left(\begin{array}{cc}
0 & -1 \\
0 & 0
\end{array}\right), \quad T^{2}=\left(\begin{array}{cc}
0 & i \\
0 & 0
\end{array}\right), \quad T^{3}=-\frac{1}{2}\left(\begin{array}{cc}
1 & 0 \\
0 & -1
\end{array}\right)
$$

in $\mathfrak{b}$. Then, the crossed product are

$$
\begin{aligned}
& \left(T_{1}, T^{1}\right)=1, \quad\left(T_{2}, T^{1}\right)=0, \quad\left(T_{3}, T^{1}\right)=0, \\
& \left(T_{1}, T^{2}\right)=0, \quad\left(T_{2}, T^{2}\right)=1, \quad\left(T_{3}, T^{2}\right)=0, \\
& \left(T_{1}, T^{3}\right)=0, \quad\left(T_{1}, T^{3}\right)=0, \quad\left(T_{3}, T^{3}\right)=1,
\end{aligned}
$$

allowing for the identification

$$
\begin{gathered}
\psi: \mathfrak{s u}_{2} \rightarrow \mathfrak{b}^{*}, \\
\psi\left(T_{1}\right)=\mathbf{t}^{1}, \quad \psi\left(T_{2}\right)=\mathbf{t}^{2}, \quad \psi\left(T_{3}\right)=\mathbf{t}^{3},
\end{gathered}
$$

where $\left\{\mathbf{t}^{1}, \mathbf{t}^{2}, \mathbf{t}^{3}\right\} \subset \mathfrak{b}^{*}$ is the dual basis to $\left\{T^{1}, T^{2}, T^{3}\right\} \subset \mathfrak{b}$. 
Let us come back to the expression (20),

$$
\begin{aligned}
\{\mathcal{F}, \mathcal{G}\}^{D}(g, \eta)= & \left\langle g \mathbf{d} \mathcal{F}, A d_{g_{-}^{-1}}^{G} \Pi_{\mathfrak{g}_{+}^{\circ}} A d_{g_{-}}^{G} \delta \mathcal{G}\right\rangle \\
& -\left\langle g \mathbf{d} \mathcal{G}, A d_{g_{-}^{-1}}^{G} \Pi_{\mathfrak{g}_{+}^{\circ}} A d_{g_{-}}^{G} \delta \mathcal{F}\right\rangle \\
& -\left\langle\eta,\left[A d_{g_{-}^{-1}}^{G} \Pi_{\mathfrak{g}_{+}^{\circ}} A d_{g_{-}}^{G} \delta \mathcal{F}, A d_{g_{-}^{-1}}^{G} \Pi_{\mathfrak{g}_{+}^{\circ}} A d_{g_{-}}^{G} \delta \mathcal{G}\right]\right\rangle
\end{aligned}
$$

and apply it to the functions

$$
\begin{aligned}
& \mathrm{T}_{i}^{j}: G \longrightarrow \mathbb{C} / \mathrm{T}_{i}^{j}(g)=g_{i}^{j}, \\
& \xi_{A}: \mathfrak{g}^{*} \longrightarrow \mathbb{C} / \xi_{A}=\left\langle\xi, \mathbb{T}_{A}\right\rangle,
\end{aligned}
$$

with

$$
\begin{aligned}
\delta \mathrm{T}_{i}^{j} & =0, \\
\mathbf{d} \xi_{A} & =0 .
\end{aligned}
$$

So, it is necessary to calculate the expressions

$$
A d_{g_{-}^{-1}}^{G} \Pi_{\mathfrak{g}_{+}^{\circ}} A d_{g_{-}}^{G} \delta \xi_{A} .
$$

The differential $\delta \xi_{A}$ coincides with the generator $\mathbb{T}_{A}$ of the Lie algebra $\mathfrak{g}$, being $\mathbb{T}_{A} \in\left\{T_{a}, T^{a}\right\}_{a=1}^{n}$. These relations can be written in terms of the coordinates for $\eta_{+}=\left\langle\eta_{+}, T_{a}\right\rangle \mathbf{t}_{a}=\xi_{a}(\eta) \mathbf{t}_{a}$ and $\eta_{-}=$ $\left\langle\eta_{-}, T^{a}\right\rangle \mathbf{t}^{a}=\xi^{a}(\eta) \mathbf{t}^{a}$. Therefore, for $A$ running on the superindex $\delta \xi_{A} \equiv \delta \xi^{a}=T^{a}$, we have $\delta \xi_{A}=$ $\delta \xi_{A-}$ and $\delta \xi_{A+}=0$. On the other side, for $A$ running on the subindex $\delta \xi_{A} \equiv \delta \xi_{a}=T_{a}$, we have $\delta \xi_{A}=\delta \xi_{A+}=\delta \xi_{a}$ and $\delta \xi_{A-}=0$. Therefore, since the only nonvanishing Dirac brackets just involve $\xi_{a}$, we evaluate

$$
A d_{g_{-}^{-1}}^{G} \Pi_{\mathfrak{g}_{+}^{\circ}} A d_{g_{-}}^{G} \delta \xi_{a}=A d_{g_{-}^{-1}}^{G} \Pi_{\mathfrak{g}_{+}^{\circ}} A d_{g_{-}}^{G} T_{a} .
$$

Writing a generic element

$$
g_{+}=\left(\begin{array}{cc}
\alpha & \beta \\
-\bar{\beta} & \bar{\alpha}
\end{array}\right) \in G_{+} \cong S U(2)
$$

and

$$
g_{-}=\left(\begin{array}{cc}
a & b+i c \\
0 & a^{-1}
\end{array}\right)=\left(\begin{array}{cc}
a & z \\
0 & a^{-1}
\end{array}\right) \in G_{-} \cong B
$$

with $a \in \mathbb{R}_{>0}, b, c \in \mathbb{R}$, we have that

$$
\begin{gathered}
A d_{g_{-}^{-1}}^{G} \Pi_{\mathfrak{g}_{+}^{\circ}} A d_{g_{-}}^{G} T_{1}=T_{1}-\left(1-\frac{b^{2}}{a^{2}}-\frac{c^{2}}{a^{2}}-\frac{1}{a^{4}}\right) T^{2}+2 \frac{c}{a} T^{3}, \\
A d_{g_{-}^{-1}}^{G} \Pi_{\mathfrak{g}_{+}^{\circ}} A d_{g_{-}}^{G} T_{2}=T_{2}+\left(1-\frac{b^{2}}{a^{2}}-\frac{c^{2}}{a^{2}}-\frac{1}{a^{4}}\right) T^{1}+2 \frac{b}{a} T^{3}, \\
A d_{g_{-}^{-1}}^{G} \Pi_{\mathfrak{g}_{+}^{\circ}} A d_{g_{-}}^{G} T_{3}=T_{3}+2 \frac{c}{a} T^{1}+2 \frac{b}{a} T^{2} .
\end{gathered}
$$

Explicit calculation for the nontrivial Dirac brackets gives

$$
\begin{aligned}
& \left\{\xi_{1}, \mathrm{~T}_{i}^{j}\right\}^{D}(g, \eta)=-\mathrm{T}_{i}^{k}(g)\left[T_{1}-\left(1-\frac{b^{2}}{a^{2}}-\frac{c^{2}}{a^{2}}-\frac{1}{a^{4}}\right) T^{2}+2 \frac{c}{a} T^{3}\right]_{k}^{j}, \\
& \left\{\xi_{2}, \mathrm{~T}_{i}^{j}\right\}^{D}(g, \eta)=-\mathrm{T}_{i}^{k}(g)\left[T_{2}+\left(1-\frac{b^{2}}{a^{2}}-\frac{c^{2}}{a^{2}}-\frac{1}{a^{4}}\right) T^{1}+2 \frac{b}{a} T^{3}\right]_{k}^{j}, \\
& \left\{\xi_{3}, \mathrm{~T}_{i}^{j}\right\}^{D}(g, \eta)=-\mathrm{T}_{i}^{k}(g)\left[T_{3}+2 \frac{c}{a} T^{1}+2 \frac{b}{a} T^{2}\right]_{k}^{j},
\end{aligned}
$$




$$
\begin{aligned}
\left\{\xi_{1}, \xi_{2}\right\}^{D}(g, \eta)=2 \frac{b}{a} \xi_{1}(\eta) & -2 \frac{c}{a} \xi_{2}(\eta)+2\left(\frac{b^{2}}{a^{2}}+\frac{c^{2}}{a^{2}}+\frac{1}{a^{4}}\right) \xi_{3}(\eta) \\
& -2 \frac{c}{a}\left(1+\frac{b^{2}}{a^{2}}+\frac{c^{2}}{a^{2}}+\frac{1}{a^{4}}\right) \xi^{1}(\eta) \\
& -2 \frac{b}{a}\left(1+\frac{b^{2}}{a^{2}}+\frac{c^{2}}{a^{2}}+\frac{1}{a^{4}}\right) \xi^{2}(\eta) \\
\left\{\xi_{1}, \xi_{3}\right\}^{D}(g, \eta)=-2 \xi_{2}(\eta)-2 \frac{c}{a} \xi_{3}(\eta) & \\
+ & 2\left(\frac{b^{2}}{a^{2}}+3 \frac{c^{2}}{a^{2}}+\frac{1}{a^{4}}-1\right) \xi^{1}(\eta) \\
+ & 4 \frac{b c}{a^{2}} \xi^{2}(\eta)+4 \frac{b}{a} \xi^{3}(\eta), \\
\left\{\xi_{2}, \xi_{3}\right\}^{D}(g, \eta)=2 \xi_{1}(\eta) & -2 \frac{b}{a} \xi_{3}(\eta)+4 \frac{b c}{a^{2}} \xi^{1}(\eta) \\
+ & 2\left(3 \frac{b^{2}}{a^{2}}+\frac{c^{2}}{a^{2}}+\frac{1}{a^{4}}-1\right) \xi^{2}(\eta)-4 \frac{c}{a} \xi^{3}(\eta)
\end{aligned}
$$

Setting $\eta_{-}=0$, the only character in $\mathfrak{b}^{*}$, they reduce to

$$
\begin{aligned}
& \left\{\xi_{1}, \mathrm{~T}_{i}^{j}\right\}^{D}(g, \eta)=-\mathrm{T}_{i}^{k}(g)\left[T_{1}\right]_{k}^{j} \\
& +\mathrm{T}_{i}^{k}(g)\left[\left(1-\frac{b^{2}}{a^{2}}-\frac{c^{2}}{a^{2}}-\frac{1}{a^{4}}\right) T^{2}+2 \frac{c}{a} T^{3}\right]_{k}^{j}, \\
& \left\{\xi_{2}, \mathrm{~T}_{i}^{j}\right\}^{D}(g, \eta)=-\mathrm{T}_{i}^{k}(g)\left[T_{2}\right]_{k}^{j} \\
& -\mathrm{T}_{i}^{k}(g)\left[\left(1-\frac{b^{2}}{a^{2}}-\frac{c^{2}}{a^{2}}-\frac{1}{a^{4}}\right) T^{1}+2 \frac{b}{a} T^{3}\right]_{k}^{j}, \\
& \left\{\xi_{3}, \mathrm{~T}_{i}^{j}\right\}^{D}(g, \eta)=-\mathrm{T}_{i}^{k}(g)\left[T_{3}\right]_{k}^{j}-\mathrm{T}_{i}^{k}(g)\left[2 \frac{c}{a} T^{1}+2 \frac{b}{a} T^{2}\right]_{k}^{j}, \\
& \left\{\xi_{1}, \xi_{2}\right\}^{D}(g, \eta)=2 \xi_{3}(\eta)+2 \frac{b}{a} \xi_{1}(\eta) \\
& -2 \frac{c}{a} \xi_{2}(\eta)-2\left(1-\frac{b^{2}}{a^{2}}-\frac{c^{2}}{a^{2}}-\frac{1}{a^{4}}\right) \xi_{3}(\eta), \\
& \left\{\xi_{3}, \xi_{1}\right\}^{D}(g, \eta)=2 \xi_{2}(\eta)+2 \frac{c}{a} \xi_{3}(\eta), \\
& \left\{\xi_{2}, \xi_{3}\right\}^{D}(g, \eta)=2 \xi_{1}(\eta)-2 \frac{b}{a} \xi_{3}(\eta) .
\end{aligned}
$$

Let us now address some dynamical model on the phase space $\mathcal{N}\left(g_{-}, 0\right)$, taking the collective Hamiltonian function

$$
\mathcal{H}(g, \eta)=-\frac{1}{16} \kappa\left(\Pi_{\mathfrak{g}_{+}^{\circ}} A d_{g}^{G} \bar{\psi}\left(\eta_{+}\right), \Pi_{\mathfrak{g}_{+}^{\circ}} A d_{g}^{G} \bar{\psi}\left(\eta_{+}\right)\right) .
$$

We may write then

$$
\mathcal{H}(g, \eta)=\frac{1}{2} \sum_{a=1}^{3} \phi^{a}(g, \eta) \phi^{a}(g, \eta) .
$$


The Hamilton equation of motion are then

$$
\begin{aligned}
& \dot{\mathrm{T}}_{j}^{k}=\left\{\mathrm{T}_{j}^{k}, \mathcal{H}\right\}^{D}, \\
& \dot{\xi}_{a}=\left\{\xi_{a}, \mathcal{H}\right\}^{D} .
\end{aligned}
$$

We may use the properties of the Poisson-Dirac bracket to get the Hamilton equation of motion

$$
\left\{\begin{array}{l}
\dot{\mathrm{T}}_{u}^{v}=\sum_{a=1}^{3} \phi^{a}\left\{\mathrm{~T}_{u}^{v}, \phi^{a}\right\}^{D} \\
\dot{\xi}_{c}=\sum_{a=1}^{3} \phi^{a}\left\{\xi_{c}, \phi^{a}\right\}^{D} .
\end{array}\right.
$$

A more explicit expression can be obtained in a compact matrix notation and considering the Hamilton equations in the form (25). In doing so, we write the Hamilton function (50) by introducing the identification $\hat{\kappa}_{\mathfrak{g}_{+}^{\circ}}: \mathfrak{g}_{+}^{\circ} \longrightarrow \mathfrak{g}_{+}^{*}$, such that $\left\langle\hat{\kappa}_{\mathfrak{g}_{+}^{\circ}}\left(X_{+}\right), Y_{+}\right\rangle=\kappa\left(X_{+}, Y_{+}\right)$, with the relations $\hat{\kappa}_{\mathfrak{g}_{+}^{\circ}}\left(T_{i}\right)$ $=-8 \mathbf{t}_{i}$, and using the bijection $(45), \bar{\psi}^{*}\left(\hat{\kappa}_{\mathfrak{g}_{+}^{\circ}}\left(T_{i}\right)\right)=-8 T^{i}$. We also introduce $\Psi: \mathfrak{g}^{*} \longrightarrow \mathfrak{g}$, which means to indicate the identification provided by the nondegenerate scalar product $(,)_{\mathfrak{g}}$ and when restricted to $\mathfrak{g}_{+}^{\circ}$ coincides with $\psi$. So that differential is

$$
\begin{aligned}
d \mathcal{H}= & (\mathbf{d} \mathcal{H}, \delta \mathcal{H}) \\
= & \left(-\frac{1}{2} \lambda R_{g^{-1}}^{*}\left(a d_{A d_{g}^{G} \bar{\Psi}(\eta)}^{\mathfrak{g} *} \hat{\kappa}_{\mathfrak{g}_{+}^{\circ}}\left(\Pi_{\mathfrak{g}_{+}^{\circ}} A d_{g}^{G} \bar{\Psi}(\eta)\right)\right),\right. \\
& \left.\frac{1}{2} \lambda A d_{g^{-1}}^{G} \bar{\Psi}^{*}\left(\hat{\kappa}_{\mathfrak{g}_{+}^{\circ}}\left(\Pi_{\mathfrak{g}_{+}^{\circ}} A d_{g}^{G} \bar{\Psi}(\eta)\right)\right)\right)
\end{aligned}
$$

and remembering that $\eta_{-}=0$, the Hamilton equations reads, for $\eta_{-}=0$,

$$
\begin{aligned}
& \left\{\begin{array}{l}
g_{+}^{-1} \dot{g}_{+}=-\frac{1}{8} \Pi_{\mathfrak{g}_{+}^{\circ}} A d_{g_{+}^{-1}}^{G} \bar{\psi}^{*}\left(\hat{\kappa}_{\mathfrak{g}_{+}^{\circ}}\left(\Pi_{\mathfrak{g}_{+}^{\circ}} A d_{g}^{G} \bar{\psi}\left(\eta_{+}\right)\right)\right) \\
A d_{g_{-}^{-1}}^{G *} \dot{\eta}_{+}=\frac{1}{8} \Pi_{\mathfrak{g}_{+}^{*}} a d_{\Pi_{\mathfrak{g}_{-}^{\circ}}^{\mathfrak{g} *} A d_{g_{+}^{-1}}^{G} \bar{\psi}^{*}\left(\hat{\kappa}_{\mathfrak{g}_{+}^{\circ}}\left(\Pi_{\mathfrak{g}_{+}^{\circ}} A d_{g}^{G} \bar{\psi}\left(\eta_{+}\right)\right)\right)}^{A d_{g_{-}^{-1}}^{G *} \eta_{+},}
\end{array}\right. \\
& \left\{\begin{array}{l}
\dot{g}_{-} g_{-}^{-1}=0 \\
\dot{\eta}_{-}=0 .
\end{array}\right.
\end{aligned}
$$

As it was shown, these nonlinear equations are solved by the $G_{+}$factor of an exponential curve in $G$, namely, $g(t)=\exp (t d \mathcal{H})$.

The model developed above comes to be a generalization of the model studied in Ref. 5, where the AKS ideas were used to provide an insight into the integrable system theory to the Poisson-Lie T-duality. This duality establishes a dynamical correspondence between Lagrangian models built on the factors of a double Lie group. To compare with those results, let us retrieve the Lagrange function associated with the above Hamiltonian system. Since,

$$
\bar{\psi}^{*}(\eta)=\eta_{1} T^{1}+\eta_{2} T^{2}+\eta_{3} T^{3}
$$

and

$$
\begin{aligned}
& g_{+}^{-1} g_{+}^{T^{3}}=\frac{1}{2} i(\alpha \bar{\beta}-\bar{\alpha} \beta) T_{1}+\frac{1}{2}(\alpha \bar{\beta}+\bar{\alpha} \beta) T_{2} \\
& g_{+}^{-1} g_{+}^{T^{2}}=-\frac{1}{2}\left(\beta^{2}+\bar{\beta}^{2}\right) T_{1}-\frac{1}{2} i\left(\beta^{2}-\bar{\beta}^{2}\right) T_{2}-\frac{1}{2}(\bar{\alpha} \bar{\beta}+\alpha \beta) T_{3} \\
& g_{+}^{-1} g_{+}^{T^{1}}=\frac{1}{2} i\left(\beta^{2}-\bar{\beta}^{2}\right) T_{1}-\frac{1}{2}\left(\beta^{2}+\bar{\beta}^{2}\right) T_{2}+\frac{1}{2} i(\alpha \beta-\bar{\alpha} \bar{\beta}) T_{3}
\end{aligned}
$$


where it was used that

$$
\bar{\psi}^{*}\left(\mathbf{t}_{1}\right)=T^{1}, \quad \bar{\psi}^{*}\left(\mathbf{t}_{2}\right)=T^{2}, \bar{\psi}^{*}\left(\mathbf{t}_{3}\right)=T^{3},
$$

we get

$$
A d_{g_{-}}^{G} \bar{\psi}\left(\eta_{+}\right)=a\left(a \eta_{+1}-b \eta_{+3}\right) T^{1}+a\left(a \eta_{+2}+c \eta_{+3}\right) T^{2}+\eta_{+3} T^{3} .
$$

Let us recall that $\phi_{X}(g, \eta)=\left\langle A d_{g^{-1}}^{G^{*}} \eta, X\right\rangle$, so that $\phi^{a}(g, \eta)=\left\langle A d_{g^{-1}}^{G^{*}} \eta_{+}, T^{a}\right\rangle$ with

$$
\begin{aligned}
\phi^{1}(g, \eta)= & \frac{1}{2} i a^{2}\left(\beta^{2}-\bar{\beta}^{2}\right) \eta_{+1}-\frac{1}{2} a^{2}\left(\beta^{2}+\bar{\beta}^{2}\right) \eta_{+2} \\
& +\frac{1}{2} i\left(\alpha \beta-\bar{\alpha} \bar{\beta}-a \bar{z} \beta^{2}+a z \bar{\beta}^{2}\right) \eta_{+3}, \\
\phi^{2}(g, \eta)= & -\frac{1}{2} a^{2}\left(\beta^{2}+\bar{\beta}^{2}\right) \eta_{+1}-\frac{1}{2} i a^{2}\left(\beta^{2}-\bar{\beta}^{2}\right) \eta_{+2} \\
& +\frac{1}{2}\left(a \bar{z} \beta^{2}+a z \bar{\beta}^{2}-\bar{\alpha} \bar{\beta}-\alpha \beta\right) \eta_{+3}, \\
\phi^{3}(g, \eta)= & -\frac{1}{2} i a^{2}(\bar{\alpha} \beta-\alpha \bar{\beta}) \eta_{+1}+\frac{1}{2} a^{2}(\alpha \bar{\beta}+\bar{\alpha} \beta) \eta_{+2} \\
& +\frac{1}{2} i a(\bar{z} \bar{\alpha} \beta-z \alpha \bar{\beta}) \eta_{+3} .
\end{aligned}
$$

Written in components, the first Hamilton equation in (51) gives rise to the differential equations

$$
\begin{aligned}
\operatorname{Im}(\bar{\alpha} \dot{\beta}-\beta \dot{\bar{\alpha}}) & =-\frac{1}{2}\left(\left(\beta^{2}+\bar{\beta}^{2}\right) \phi^{2}-i\left(\beta^{2}-\bar{\beta}^{2}\right) \phi^{1}-i(\alpha \bar{\beta}-\bar{\alpha} \beta) \phi^{3}\right), \\
\operatorname{Re}(\bar{\alpha} \dot{\beta}-\beta \dot{\bar{\alpha}}) & =-\frac{1}{2}\left(\left(\beta^{2}+\bar{\beta}^{2}\right) \phi^{1}+i\left(\beta^{2}-\bar{\beta}^{2}\right) \phi^{2}-(\alpha \bar{\beta}+\bar{\alpha} \beta) \phi^{3}\right), \\
(\bar{\alpha} \dot{\alpha}+\beta \dot{\bar{\beta}}) & =-\frac{1}{2}\left((\bar{\alpha} \bar{\beta}+\alpha \beta) \phi^{2}-i(\alpha \beta-\bar{\alpha} \bar{\beta}) \phi^{1}\right) .
\end{aligned}
$$

We use these equations to invert the Legendre transformation in order to retrieve the Lagrange function. From these equations, we write $\eta_{+1}$ and $\eta_{+2}$ in terms of the velocities $\bar{\alpha} \dot{\beta}-\beta \dot{\bar{\alpha}}$ and $\bar{\alpha} \dot{\alpha}+\beta \bar{\beta}$. Thus, the expression of the momentum map associated with the dressing action are

$$
\begin{aligned}
{\left[\Pi_{\mathfrak{g}_{+}^{\circ}} A d_{g}^{G} \bar{\psi}\left(\eta_{+}\right)\right]_{1}=} & -\frac{1}{2 \beta \bar{\alpha}}(\alpha \beta+\bar{\beta} \bar{\alpha})(\bar{\alpha} \dot{\beta}-\beta \dot{\bar{\alpha}}) \\
& +\frac{1}{2 \bar{\alpha}|\beta|^{4}} \beta\left(|\beta|^{4}+|\beta|^{2}-\bar{\alpha}^{2} \bar{\beta}^{2}\right)(\bar{\alpha} \dot{\alpha}+\beta \dot{\bar{\beta}}), \\
{\left[\Pi_{\mathfrak{g}_{+}^{\circ}} A d_{g}^{G} \bar{\psi}\left(\eta_{+}\right)\right]_{2}=} & -i \frac{1}{2 \beta \bar{\alpha}}(\alpha \beta-\bar{\beta} \bar{\alpha})(\bar{\alpha} \dot{\beta}-\beta \dot{\bar{\alpha}}) \\
& +i \frac{1}{2 \bar{\alpha}|\beta|^{4}} \beta\left(\bar{\alpha}^{2} \bar{\beta}^{2}+|\beta|^{4}+|\beta|^{2}\right)(\bar{\alpha} \dot{\alpha}+\beta \dot{\bar{\beta}}), \\
{\left[\Pi_{\mathfrak{g}_{+}^{\circ}} A d_{g}^{G} \bar{\psi}\left(\eta_{+}\right)\right]_{3}=} & \frac{\alpha}{\beta}(\bar{\alpha} \dot{\beta}-\beta \dot{\bar{\alpha}})-(\bar{\alpha} \dot{\alpha}+\beta \overline{\bar{\beta}}),
\end{aligned}
$$


where we may observe that there are no trace of $g_{-}$. So, the expression for the Hamilton function in terms of the velocities is the same in each phase space $\mathcal{N}\left(g_{-}, 0\right)$,

$$
\begin{aligned}
& \mathcal{H}(g, \dot{g}) \\
= & \frac{1}{2|\beta|^{2}}\left(\frac{\alpha \bar{\beta}}{\bar{\alpha} \beta}(\bar{\alpha} \dot{\beta}-\beta \dot{\bar{\alpha}})^{2}-2 \frac{\bar{\beta}}{\bar{\alpha}}(\bar{\alpha} \dot{\alpha}+\beta \dot{\bar{\beta}})(\bar{\alpha} \dot{\beta}-\beta \dot{\bar{\alpha}})-(\bar{\alpha} \dot{\alpha}+\beta \dot{\bar{\beta}})^{2}\right) .
\end{aligned}
$$

Let us then built up the Lagrangian function describing the dynamics of these systems. It is obtained as usual

$$
L_{\mathcal{N}\left(g_{-}, 0\right)}(g, \dot{g})=\left\langle\eta_{+}, g_{+}^{-1} \dot{g}_{+}\right\rangle-\mathcal{H}\left(g_{+}, \eta_{+}\right)
$$

where

$$
\left\langle\eta_{+}, g_{+}^{-1} \dot{g}_{+}\right\rangle=\eta_{+1} \operatorname{Im}(\bar{\alpha} \dot{\beta}-\beta \dot{\bar{\alpha}})+\eta_{+2} \operatorname{Re}(\bar{\alpha} \dot{\beta}-\beta \dot{\bar{\alpha}})-i \eta_{+3}(\bar{\alpha} \dot{\alpha}+\beta \dot{\bar{\beta}}) .
$$

After some tedious calculations, we get

$$
\begin{aligned}
& L_{\mathcal{N}\left(g_{-}, 0\right)}\left(g_{+}, \dot{g}_{+}\right) \\
= & \frac{1}{2 a^{2}|\beta|^{2}}|\bar{\alpha} \dot{\beta}-\beta \dot{\bar{\alpha}}|^{2}+\frac{1}{2|\beta|^{2}} \frac{\alpha \bar{\alpha}}{\bar{\alpha} \beta}\left(\frac{1}{a^{2}}-1\right)(\bar{\alpha} \dot{\beta}-\beta \dot{\bar{\alpha}})^{2} \\
& +\frac{1}{2 a^{2}|\beta|^{2}} \frac{\bar{\alpha}}{\bar{\alpha}}\left(2 a^{2}-1-\frac{1}{|\beta|^{2}}\right)(\bar{\alpha} \dot{\beta}-\beta \dot{\bar{\alpha}})(\bar{\alpha} \dot{\alpha}+\beta \dot{\bar{\beta}}) \\
& +\frac{1}{2 a^{2}|\beta|^{4}} \beta \bar{\alpha}(\bar{\alpha} \dot{\alpha}+\beta \dot{\bar{\beta}}) \overline{(\bar{\alpha} \dot{\beta}-\beta \dot{\bar{\alpha}})}+\frac{1}{2|\beta|^{2}}(\bar{\alpha} \dot{\alpha}+\beta \dot{\bar{\beta}})^{2} \\
& +\eta_{+3} \frac{1}{2 a^{2}|\beta|^{2}}\left(2 \operatorname{Im}(\bar{\beta}(a \bar{z} \beta-\alpha)(\bar{\alpha} \dot{\beta}-\beta \dot{\bar{\alpha}}))-2 i a^{2}|\beta|^{2}(\bar{\alpha} \dot{\alpha}+\beta \dot{\bar{\beta}})\right) .
\end{aligned}
$$

Observe that the last term contains the Lagrange multiplier $\eta_{+3}$ realizing the constraint

$$
\begin{aligned}
& \Omega\left(g_{+}, g_{-}\right) \\
= & i \beta(a z \bar{\beta}-\bar{\alpha}) \overline{(\bar{\alpha} \dot{\beta}-\beta \dot{\bar{\alpha}})}-\bar{\beta}(a \bar{z} \beta-\alpha)(\bar{\alpha} \dot{\beta}-\beta \dot{\bar{\alpha}})-2 i a^{2}|\beta|^{2}(\bar{\alpha} \dot{\alpha}+\beta \dot{\bar{\beta}}) .
\end{aligned}
$$

Despite its rather complicated expression, this Lagrange function can be written in the compact form

$$
L_{\mathcal{N}\left(g_{-}, 0\right)}\left(g_{+}, \dot{g}_{+}\right)=-\frac{1}{8} \kappa\left(g_{+}^{-1} \dot{g}_{+}, \mathbb{K}\left(g_{+}, g_{-}\right) g_{+}^{-1} \dot{g}_{+}\right)-\lambda \Omega\left(g_{+}, g_{-}\right),
$$

where $\lambda$ is a redefinition of the Lagrange multiplier, and $\mathbb{K}\left(g_{+}, g_{-}\right): \mathfrak{s u}_{2} \longrightarrow \mathfrak{s u}_{2}$ is a bijection that in the basis $\left\{T_{1}, T_{2}, T_{3}\right\}$ is represented by the symmetric matrix, a metric tensor,

$$
\mathbb{K}\left(g_{+} g_{-}\right)=\frac{1}{2|\beta|^{2}}\left(\begin{array}{ccc}
1 & 0 & \mathrm{~m} \\
0 & 1 & \mathrm{n} \\
\mathrm{m} & \mathrm{n} & 1
\end{array}\right),
$$

where

$$
\begin{aligned}
& \mathrm{m}=\frac{\left(\left(a^{2}-1\right)(\beta \bar{\alpha}+\alpha \bar{\beta})+a(\bar{z}+z) \beta \bar{\beta}\right)}{2 a^{2} \beta \bar{\beta}}, \\
& \mathrm{n}=i \frac{\left(\left(1-a^{2}\right)(\alpha \bar{\beta}-\beta \bar{\alpha})+a(z-\bar{z}) \beta \bar{\beta}\right)}{2 a^{2} \beta \bar{\beta}} .
\end{aligned}
$$


It is worth to remark that $g_{-}=e$, it turns in

$$
\mathbb{K}=\frac{1}{2|\beta|^{2}}\left(\begin{array}{lll}
1 & 0 & 0 \\
0 & 1 & 0 \\
0 & 0 & 1
\end{array}\right),
$$

thus recovering the Lagrangian function described in Ref. 5.

The constraint $\Omega$ can be written as

$$
\Omega=\kappa\left(\mathbb{A}\left(g_{+} g_{-}\right), g_{+}^{-1} \dot{g}_{+}\right)
$$

for

$$
\begin{aligned}
\mathbb{A}\left(g_{+}, g_{-}\right)= & \frac{1}{8} i(\beta(a z \bar{\beta}-\bar{\alpha})+\bar{\beta}(a \bar{z} \beta-\alpha)) T_{1} \\
& -\frac{1}{8}(\beta(a z \bar{\beta}-\bar{\alpha})-\bar{\beta}(a \bar{z} \beta-\alpha)) T_{2}+\frac{1}{4} a^{2} \beta \bar{\beta} T_{3},
\end{aligned}
$$

so that the velocities must be confined in the orthogonal complement of the vector $\mathbb{A}(g b)$. The Lagrangian function is now written as

$$
L_{\mathcal{N}\left(g_{-}, 0\right)}\left(g_{+}, \dot{g}_{+}\right)=-\frac{1}{8} \kappa\left(g_{+}^{-1} \dot{g}_{+}, \mathbb{K}\left(g_{+} g_{-}\right) g_{+}^{-1} \dot{g}_{+}\right)-\frac{1}{8} \lambda \kappa\left(\mathbb{A}\left(g_{+}, g_{-}\right), g_{+}^{-1} \dot{g}_{+}\right)_{\mathfrak{g}_{+}^{\circ}},
$$

where $\mathbb{A}(g b) \in \mathfrak{g}$ was given in (53). It is equivalent to

$$
L_{\mathcal{N}\left(g_{-}, 0\right)}\left(g_{+}, \dot{g}_{+}\right)=-\frac{1}{8} \kappa\left(g_{+}^{-1} \dot{g}_{+}, \mathbb{K}\left(g_{+} g_{-}\right) g_{+}^{-1} \dot{g}_{+}+\lambda \mathbb{A}\left(g_{+}, g_{-}\right)\right) .
$$

Then, we conclude that the change at the Lagrangian level introduced by moving away from the fiber on $(e, 0)$ amounts to a nontrivial metric on the configuration space associated with $\mathcal{N}\left(g_{-}, 0\right)$, where the parameters of $g_{-}$regulate the coupling. Since this example is motivated by the study developed in Ref. 5, where it was shown that the system on the fiber $\mathcal{N}\left(g_{-}, 0\right)$ is T-dual related to the Toda model, it remains as an interesting problem to find the class of T-dual systems associated with those on the fibers for generic $\left(g_{-}, 0\right)$.

\section{CONCLUSIONS}

Using the Dirac method, we have studied a class of symplectic submanifolds in the cotangent bundle of a factorizable Lie group. They appear as generalizations of the cotangent bundles of the factors, in fact, they are recovered when the fibers at $(e, 0) \in G \times \mathfrak{g}^{*}$ are considered. These fibrations amount to be a symplectic one: any horizontal curve is a symplectomorphism between fibers. This analysis leads to some remarkable results about these reduced phase spaces, and a versatile interplay between their symplectic geometry and the Poisson algebra of functions on the whole phase space.

A suitable formulation of the Dirac procedure allows us to describe the restriction of the Lie algebra of functions on $G \times \mathfrak{g}^{*}$ to the submanifolds $\mathcal{N}\left(g_{-}, \eta_{-}\right)$and $\mathcal{M}\left(g_{+}, \eta_{+}\right)$in a natural fashion, showing how symmetries and dynamics projects from the big phase space to the smaller ones. This gives rise to the following remarkable fact: Dirac brackets of the momentum functions associated with the let translations in $G \times \mathfrak{g}^{*}$ give rise to actions of $G$ on $\mathcal{N}\left(g_{-}, \eta_{-}\right)$and $\mathcal{M}\left(g_{+}, \eta_{+}\right)$provided $\eta_{-}$and $\eta_{+}$, respectively, are characters of the corresponding coadjoint actions of $G_{-}$and $G_{+}$. So these special fibers are orbits of an action of $G$ which is not free, in general.

It also entails the most relevant result of this work, namely, the realization of the AKS ideas through the Dirac method. In fact, these actions play a central role in the connection with integrable systems: collective dynamics built with these momentum functions on $G \times \mathfrak{g}^{*}$, give rise to Dirac Hamiltonian vector fields which are of the AKS type, being integrable in this sense. The framework developed above turns the factorization problem in a constrained system one, where the Dirac method becomes a natural tool for dealing with. We have shown that Dirac brackets nicely reduce a somehow trivial systems on $G \times \mathfrak{g}^{*}$ into a system with plenty of nontrivial dynamics. So, we may say that Dirac method naturally implements the idea of solving some nonlinear systems as a part of a simpler system on a bigger phase space. 
A deeper geometric insight, besides a description of the AKS systems suitable for our purposes, allowed us to explain this (apparently) unexpected connection between Dirac constraints and integrable AKS system, as arising from the description of the latter as reduced space of $T^{*} G$, and by the existence of immersions of the cotangent bundle of some homogeneous spaces of $G$ into $T^{*} G$.

In the examples, it was shown that the standard symplectic forms are recovered when one of the factors is chosen as a trivial space and, also that there are nontrivial modifications of the dynamical systems in passing from one fiber to another. The example on $S L(2, \mathbb{C}) \cong S U(2) \times B$ exhibits a hard coupling with a metric on the associated configuration space which disappears for the fiber at $(e, 0)$, the cotangent bundle of $S U(2)$.

As a final remark, we conclude that the Dirac procedure applied to both the families of symplectic submanifolds in $G \times \mathfrak{g}^{*}$ provides a way to produce the AKS integrable systems. The approach presented above assembles double Lie groups and the Dirac theory of constraints to encode successfully the ideas of AKS integrable systems.

\section{ACKNOWLEDGMENTS}

The authors thank to CONICET (Argentina) for financial support.

${ }^{1}$ Adler, M. and van Moerbeke, P., "Completely integrable systems, Euclidean Lie algebras and curves," Adv. Math. 38, 267-317 (1980); Kostant, B., "The solution to a generalized Toda lattice and representation theory," ibid. 34, 195-338 (1979); Symes, W., "Systems of Toda type, inverse spectral problem and representation theory," Invent. Math. 159, 13-51 (1980).

2 Ahbraham, M. and Marsden, J., Foundations of Mechanics, 2nd ed. (Benjamin/Cummings, Reading, MA, 1978).

${ }^{3}$ Arnold, V. I., Mathematical Methods of Classical Mechanics (Springer-Verlag, New York, 1989).

${ }^{4}$ Cabrera, A. and Montani, H., "Hamiltonian loop group actions and T-Duality for group manifolds," J. Geom. Phys. 56, 1116-1143 (2006); e-print hep-th/0412289.

${ }^{5}$ Capriotti, S. and Montani, H., "Integrable systems and Poisson-Lie T-duality: A finite dimensional example," J. Geom. Phys. 60, 1509-1529 (2010); e-print arXiv:math-phys/0910.3236v1.

${ }^{6}$ Dirac, P. A. M., Lectures on Quantum Mechanics, Belfer Graduate School of Science Monographs Series Vol. 2 (Yeshiva University, New York, 1964).

${ }^{7}$ Lu, J.-H. and Weinstein, A., "Poisson Lie groups, dressing transformations, and Bruhat decompositions," J. Differential Geom. 31, Number 2, 501-526 (1990).

${ }^{8}$ Marsden, J. E., Misiolek, G., Perlmutter, M., and Ratiu, T. S., "Symplectic reduction for semidirect products and central extensions," Diff. Geom. Appl. 9, 173-212 (1998).

${ }^{9}$ Marsden, J. and Ratiu, T., Introduction to Mechanics and Symmetry, 2nd ed. (Benjamin/Cummings, Reading, MA, 1978).

${ }^{10}$ Marsden, J. E. and Weinstein, A., "Reduction of symplectic manifolds with symmetry," Rep. Math. Phys. 5, 121-131 (1974).

${ }^{11}$ Reyman, A. G. and Semenov-Tian-Shansky, M. A., "Reduction of Hamiltonian systems, affine Lie algebras, and Lax equations I," Invent. Math. 54, 81-100 (1979); "Reduction of Hamiltonian systems, affine Lie algebras, and Lax equations II," ibid 63, 423-32 (1981).

${ }^{12}$ Reyman, A. G. and Semenov-Tian-Shansky, M. A., "Group-theoretical methods in the theory of finite-dimensional integrable systems," in Dynamical Systems VII (Encyclopaedia of Mathematical Sciences), edited by V. I. Arnold and S. P. Novikov (Springer, New York, 1993).

${ }^{13}$ Semenov-Tian-Shansky, M. A., "Dressing transformations and Poisson group actions," Publ. RIMS, Kyoto Univ. 21, 1237-1260 (1985). 